ESAIM: COCV 21 (2015) 535-560

DOI: $10.1051 / \mathrm{cocv} / 2014037$
ESAIM: Control, Optimisation and Calculus of Variations

www.esaim-cocv.org

\title{
COUPLING ESTIMATION AND CONTROL FOR A TWO DIMENSIONAL BURGERS TYPE EQUATION *
}

\author{
JeAn-Marie Buchot ${ }^{1}$, Jean-Pierre Raymond ${ }^{2}$ And Jorge Tiago ${ }^{3}$
}

\begin{abstract}
The aim of this paper is to study the boundary feedback stabilization of a two dimensional Burgers type equation with a Dirichlet boundary control and boundary measurements. Thus we have to deal with highly unbounded control and observation operators. We study the well posedness of the infinite dimensional system obtained by coupling a linear estimator with a linear feedback control law for the corresponding linearized parabolic system in a neighborhood of an unstable stationary solution. We prove the local stabilization of the system obtained by applying to the nonlinear equation the linear feedback control coupled with the linear compensator. Numerical experiments confirm the theoretical results.
\end{abstract}

Mathematics Subject Classification. 93B52, 93C20, 93E10.

Received June 12, 2013. Revised June 26, 2014

Published online March 9, 2015.

\section{INTRODUCTION}

We are interested in coupling a feedback control law and a state estimation based on boundary measurements for a $2 \mathrm{D}$ Burgers type equation. The two dimensional domain $\Omega$ in which we consider the Burgers equation is the rectangle $\Omega=\left(0, L_{1}\right) \times\left(0, L_{2}\right)$, with $L_{1}>0$ and $L_{2}>0$. Its boundary $\Gamma$ is split into two parts. Dirichlet boundary conditions are prescribed on $\Gamma_{d}=\left[0, L_{1}\right] \times\{0\}$, while Neumann boundary conditions are imposed on $\Gamma_{n}=\Gamma \backslash \Gamma_{d}$. The system is controlled by a Dirichlet control of finite dimension, prescribed on $\Gamma_{d}$, and it is subject to disturbances of finite dimension in the Neumann boundary conditions. More precisely, we consider

\footnotetext{
Keywords and phrases. Burgers equation, feedback law, estimation, boundary control, compensator, boundary measurements, semilinear parabolic equations.

* The first two authors were partially supported by the ANR-project CISIFS 09-BLAN-0213-03 and the FNRAE-project ECOSEA. The third author was supported by Fundação para a Ciência e a Tecnologia (Portugal), postdoctoral grant SFRHBPD-66639-2009 FCT POPH - FSE.

1 Institut de Mathématiques de Toulouse, UMR CNRS 5219, Université Paul Sabatier, 31062 Toulouse cedex 9, France. jean-marie.buchot@math.univ-toulouse.fr

2 Institut de Mathématiques de Toulouse, UMR CNRS 5219, Université Paul Sabatier, 31062 Toulouse cedex 9, France. jean-pierre.raymond@math.univ-toulouse.fr

3 CEMAT-IST, Lisbon, Portugal. jftiago@math.ist.utl.pt
} 
the following partial differential equation

$$
\begin{aligned}
& \frac{\partial w}{\partial t}-\nu \Delta w+\left(\partial_{1} w+\partial_{2} w\right) w=f_{s} \quad \text { in } \Omega \times(0, \infty), \\
& \nu \frac{\partial w}{\partial n}=h_{s}+\sum_{i=1}^{N_{d}} \zeta_{i} h_{i} \quad \text { on } \Gamma_{n} \times(0, \infty), \quad w=g_{s}+\sum_{i=1}^{N_{c}} u_{i} g_{i} \quad \text { on } \Gamma_{d} \times(0, \infty), \\
& w(0)=w_{0}+\mu_{0} \quad \text { in } \Omega .
\end{aligned}
$$

In this setting, $\nu$ is a positive constant, for $j=1$ or $j=2, \partial_{j}$ stands for $\frac{\partial}{\partial x_{j}}, f_{s}, g_{s}, h_{s},\left(g_{i}\right)_{1 \leq i \leq N_{c}}$ and $\left(h_{i}\right)_{1 \leq i \leq N_{d}}$ are stationary data (independent of $t$ ), while $u=\left(u_{1}, \ldots, u_{N_{c}}\right) \in L^{2}\left(0, \infty ; \mathbb{R}^{N_{c}}\right)$ is the control variable depending only on $t, \mu_{0}$ is an uncertainty in the initial condition, $\sum_{i=1}^{N_{d}} \zeta_{i} h_{i}$ is a disturbance model in a boundary condition, $\zeta=\left(\zeta_{1}, \ldots, \zeta_{N_{d}}\right) \in H^{1}\left(0, \infty ; \mathbb{R}^{N_{d}}\right)$. We assume that $g_{i} \in H^{3 / 2}\left(\Gamma_{d}\right) \cap H_{0}^{1}\left(\Gamma_{d}\right)$ for $1 \leq i \leq N_{c}$, and that $h_{j} \in H^{3 / 2}\left(\Gamma_{n}\right)$ for $1 \leq j \leq N_{d}$.

We denote by $w_{s}$ a solution in $H^{2}(\Omega)$ to the following stationary equation

$$
\begin{aligned}
-\nu \Delta w_{s}+\left(\partial_{1} w_{s}+\partial_{2} w_{s}\right) w_{s} & =f_{s} \quad \text { in } \Omega, \\
\nu \frac{\partial w_{s}}{\partial n} & =h_{s} \quad \text { on } \Gamma_{n}, \quad w_{s}=g_{s} \quad \text { on } \Gamma_{d} .
\end{aligned}
$$

We are interested in the local feedback stabilization of equation (1.1) around the stationary solution $w_{s}$, with a prescribed exponential decay rate $-\omega$, in the case of partial information. This means that we look for a control $u$ of the form

$$
u(t)=K\left(w_{e}(t)-w_{s}\right),
$$

where $w_{e}$ is an estimation of the state variable $w$, such that the solution $w$ of equation (1.1) corresponding to $u$ defined by (1.3) obeys

$$
\left\|\mathrm{e}^{\omega t}\left(w(t)-w_{s}\right)\right\|_{H^{\varepsilon}(\Omega)} \leq \vartheta\left(\left\|w_{0}\right\|_{H^{\varepsilon}(\Omega)}\right) \quad \text { for all } t \geq 0
$$

provided that $\left\|w_{0}-w_{s}\right\|_{H^{\varepsilon}(\Omega)}$ is small enough for some $\varepsilon>0$ (here $\vartheta\left(\left\|w_{0}\right\|_{H^{\varepsilon}(\Omega)}\right)$ denotes a function of $\left.\left\|w_{0}\right\|_{H^{\varepsilon}(\Omega)}\right)$.

It is convenient to write the nonlinear equation satisfied by $v=w-w_{s}$ :

$$
\begin{aligned}
& \frac{\partial v}{\partial t}-\nu \Delta v+\left(\partial_{1} w_{s}+\partial_{2} w_{s}\right) v+\left(\partial_{1} v+\partial_{2} v\right) w_{s}+\left(\partial_{1} v+\partial_{2} v\right) v=0 \quad \text { in } \Omega \times(0, \infty), \\
& \nu \frac{\partial v}{\partial n}=\sum_{i=1}^{N_{d}} \zeta_{i} h_{i} \quad \text { on } \Gamma_{n} \times(0, \infty), \quad v=\sum_{i=1}^{N_{c}} u_{i} g_{i} \quad \text { on } \Gamma_{d} \times(0, \infty), \\
& v(0)=w_{0}+\mu_{0}-w_{s}=v_{0}+\mu_{0} \quad \text { in } \Omega .
\end{aligned}
$$

Next, we denote by $w_{\zeta}(t)$ the solution to the equation

$$
\begin{aligned}
& \lambda_{0} w_{\zeta}(t)-\nu \Delta w_{\zeta}(t)+\left(\partial_{1} w_{s}+\partial_{2} w_{s}\right) w_{\zeta}(t)+\left(\partial_{1} w_{\zeta}(t)+\partial_{2} w_{\zeta}(t)\right) w_{s}=0 \quad \text { in } \Omega, \\
& \nu \frac{\partial w_{\zeta}(t)}{\partial n}=\sum_{i=1}^{N_{d}} \zeta_{i}(t) h_{i} \quad \text { on } \Gamma_{n}, \quad w_{\zeta}(t)=0 \quad \text { on } \Gamma_{d},
\end{aligned}
$$


where $\lambda_{0}>0$ is chosen large enough so that (2.3) is satisfied. If $\zeta=\left(\zeta_{i}\right)_{1 \leq i \leq N_{d}} \in H^{1}\left(0, \infty ; \mathbb{R}^{N_{d}}\right)$, we can verify that $z=v-w_{\zeta}$ satisfy the following equation

$$
\begin{aligned}
& \frac{\partial z}{\partial t}-\nu \Delta z+\left(\partial_{1} w_{s}+\partial_{2} w_{s}\right) z+\left(\partial_{1} z+\partial_{2} z\right) w_{s}+\left(\partial_{1}\left(w_{\zeta}+z\right)+\partial_{2}\left(w_{\zeta}+z\right)\right)\left(w_{\zeta}+z\right)=\mu \\
& \text { in } \Omega \times(0, \infty), \\
& \nu \frac{\partial z}{\partial n}=0 \quad \text { on } \Gamma_{n} \times(0, \infty), \quad z=\sum_{i=1}^{N_{c}} u_{i} g_{i} \quad \text { on } \Gamma_{d} \times(0, \infty), \\
& z(0)=v_{0}+\mu_{0}-w_{\zeta}(0)=z_{0}+\mu_{0} \quad \text { in } \Omega,
\end{aligned}
$$

with $\mu=-\frac{\partial w_{\zeta}}{\partial t}+\lambda_{0} w_{\zeta}$. The corresponding linearized equation is

$$
\begin{aligned}
& \frac{\partial z}{\partial t}-\nu \Delta z+\left(\partial_{1} w_{s}+\partial_{2} w_{s}\right) z+\left(\partial_{1} z+\partial_{2} z\right) w_{s}=\mu \quad \text { in } \Omega \times(0, \infty), \\
& \nu \frac{\partial z}{\partial n}=0 \quad \text { on } \Gamma_{n} \times(0, \infty), \quad z=\sum_{i=1}^{N_{c}} u_{i} g_{i} \quad \text { on } \Gamma_{d} \times(0, \infty), \\
& z(0)=z_{0}+\mu_{0} \quad \text { in } \Omega .
\end{aligned}
$$

In Section 2, we shall explain that equation (1.6) may be rewritten in the form

$$
z^{\prime}=A z+F\left(w_{\zeta}+z\right)+B u+\mu, \quad z(0)=z_{0}+\mu_{0},
$$

where $A$, with domain $D(A)$ in $L^{2}(\Omega)$, is the infinitesimal generator of an analytic semigroup on $L^{2}(\Omega)$, and $B$ is a bounded operator from $\mathbb{R}^{N_{c}}$ into $\left(\mathcal{D}\left(A^{*}\right)\right)^{\prime}$.

We shall choose some measurements depending on $\left.w(t)\right|_{\Gamma_{n}}$ or on $\left.z(t)\right|_{\Gamma_{n}}$, of the form

$$
H z(t)=\left(\frac{1}{\left|\Gamma_{1}\right|} \int_{\Gamma_{1}} z(t), \ldots, \frac{1}{\left|\Gamma_{N_{o}}\right|} \int_{\Gamma_{N_{o}}} z(t)\right) \in \mathbb{R}^{N_{o}},
$$

where $\Gamma_{1}, \ldots, \Gamma_{N_{o}}$ are nonempty open intervals in $\Gamma_{n}$.

We have already studied similar problems, in the case of full information, for the Burgers equation in [38], and for the Navier-Stokes equations in [31,32,34]. In [31,34], the feedback control law is determined by stabilizing the linearized model and this feedback law is next applied to the nonlinear system. Here we are going to follow the same approach but in the case of partial information.

We denote by $Z=L^{2}(\Omega)$ the state space, by $U=\mathbb{R}^{N_{c}}$ the control space and by $Y=\mathbb{R}^{N_{o}}$ the observation space. In Section 2, we shall see that the following assumptions are satisfied.

$\left(H_{1}\right)$ The operator $A$, with domain $\mathcal{D}(A)$, is the infinitesimal generator of an analytic semigroup on $Z$. The resolvent of $A$ is compact.

$\left(H_{2}\right)$ The operator $B \in \mathcal{L}\left(U,\left(\mathcal{D}\left(A^{*}\right)\right)^{\prime}\right)$ satisfies

$$
\left(\lambda_{0} I-A\right)^{-\alpha} B \in \mathcal{L}(U, Z), \quad \text { for some } 0 \leq \alpha<1,
$$

where $\lambda_{0}>0$ is chosen so that $\lambda_{0} I-A$ is a positive operator.

$\left(H_{3}\right)$ The operator $H$ is defined in $\mathcal{D}(H)$, a subspace of $Z$. We assume that $\mathcal{D}(A) \subset \mathcal{D}(H)$ and that

$$
H\left(\lambda_{0} I-A\right)^{-\beta} \in \mathcal{L}(Z, Y), \quad \text { for some } 0 \leq \beta<1 .
$$

Our goal is to find $K \in \mathcal{L}(Z, U)$ and $L \in \mathcal{L}(Y, Z)$ such that the system

$$
\begin{aligned}
& z^{\prime}=A z+F\left(w_{\zeta}+z\right)+B K z_{e}+\mu, \quad z(0)=z_{0}+\mu_{0}, \\
& z_{e}^{\prime}=A z_{e}+B K z_{e}+L\left(H z_{e}-y_{o b s}\right), \quad z_{e}(0)=z_{0}, \\
& y_{o b s}(t)=H z(t)+\eta(t),
\end{aligned}
$$


is well posed and that its solution $\left(z, z_{e}\right)$ satisfies

$$
\left\|\mathrm{e}^{\omega t} z(t)\right\|_{Z}+\left\|\mathrm{e}^{\omega t} z_{e}(t)\right\|_{Z} \longrightarrow 0 \quad \text { as } \quad t \longrightarrow \infty
$$

for some $\omega>0$, provided that $z_{0}, \mu_{0}, \mu$ and $\eta$ are small enough in appropriate norms.

Since we want to deal with systems corresponding to boundary controls and boundary measurements, we are interested in the case when $\alpha+\beta>1$. To the best of our knowledge, there is no result in the literature on the feedback stabilization with partial information of nonlinear systems of the form (1.8) in the case when $\alpha+\beta>1$.

The local stabilization result for system (1.12) will be obtained by determining $K \in \mathcal{L}(Z, U)$ and $L \in \mathcal{L}(Y, Z)$ such that the solution $\left(z, z_{e}\right)$ to the linearized system

$$
\begin{aligned}
z^{\prime} & =A z+B K z_{e}+\mu, \quad z(0)=z_{0}+\mu_{0}, \\
z_{e}^{\prime} & =A z_{e}+B K z_{e}+L\left(H z_{e}-y_{o b s}\right), \quad z_{e}(0)=z_{0}, \\
y_{o b s}(t) & =H z(t)+\eta(t),
\end{aligned}
$$

with $z_{0} \in Z$ and $\mu_{0} \in Z$, obeys (1.13) provided that $\mu$ and $\eta$ are bounded in appropriate norms. In order that the solution $\left(z, z_{e}\right)$ to system (1.14) obeys (1.13), we have to prove (or to assume) that

$$
(A+\omega I, B) \text { is stabilizable and }(A+\omega I, H) \text { is detectable. }
$$

The equation

$$
z_{e}^{\prime}=A z_{e}+B K z_{e}+L\left(H z_{e}-y_{o b s}\right), \quad z_{e}(0)=z_{0}
$$

is called 'the estimation equation' or the 'compensator'. It is the equation used to determine the control $B K z_{e}$ involved in the system satisfied by $z$.

Schumacher [36], Curtain [11], and Curtain and Salamon [13] have proposed finite dimensional compensators of the form $(1.14)_{2}$ for linear systems in the case when $\alpha+\beta \leq 1 / 2$. One way to find a finite dimensional compensator consists in using a finite dimensional approximation of equation (1.15). This is the way followed by Lasiecka [25] in the case when $\alpha+\beta<1$, and next by Ji and Lasiecka [20] in the case when $\alpha+\beta>1$.

In pratical applications, it is convenient to have a compensator of finite dimension. It is not the case of equation (1.15). Following [20], we use a finite element approximation in our numerical simulations. Our future goal will be to construct a finite dimensional estimator of small dimension. But for the applications we have in view (stabilization of fluid flows), an estimator based on the approximation of $A$ by a finite element method (or another classical approximation method) leads to a finite dimensional estimator of too large dimension to be useful. We shall present in a future work new estimators of finite dimension. In Section 2.4, we are going to see that the assumptions $\left(H_{1}\right)-\left(H_{3}\right)$ are not sufficient to establish the well posedness of equation $(1.14)_{2}$.

Additional references on compensators for infinite dimensional systems are $[4,7,12,24]$.

The plan of the paper is as follows. We study the feedback control law and the estimation equation for the linearized Burgers equation (1.7) in Section 2. The local stabilization of the nonlinear system (1.12) is studied in Section 3. In Section 4, we introduce the finite element approximation used in the numerical tests. Some numerical experiments, in which we test the efficiency of the compensator both for the linearized Burgers equation and for the corresponding nonlinear model, are provided in Section 5.

\section{FEEDBACK LAW AND FILTERING OPERATOR}

The aim of this section is to study the feedback law and the estimation of the linearized Burgers equation (1.7). 


\subsection{Assumptions and preliminary results for the Burgers equation}

In order to write equation (1.7) as a controlled system, we set

$$
\mathcal{D}(A)=\left\{z \in H^{2}(\Omega) \mid z=0 \text { on } \Gamma_{d} \text { and } \frac{\partial z}{\partial n}=0 \text { on } \Gamma_{n}\right\},
$$

and

$$
A z=\nu \Delta z-\left(\partial_{1} w_{s}+\partial_{2} w_{s}\right) z-\left(\partial_{1} z+\partial_{2} z\right) w_{s} \text { for all } z \in \mathcal{D}(A) .
$$

Since $w_{s} \in H^{2}(\Omega)$, it is clear that $A z \in Z=L^{2}(\Omega)$ when $z \in \mathcal{D}(A)$. We also introduce the operator $A_{0}$ corresponding to the particular case when $w_{s}=0$ :

$$
\mathcal{D}\left(A_{0}\right)=\left\{z \in H^{2}(\Omega) \mid z=0 \text { on } \Gamma_{d} \text { and } \frac{\partial z}{\partial n}=0 \text { on } \Gamma_{n}\right\},
$$

and

$$
A_{0} z=\nu \Delta z \quad \text { for all } z \in \mathcal{D}\left(A_{0}\right) .
$$

Theorem 2.1. The operator $(A, \mathcal{D}(A))$ defined in $(2.1)$ is the infinitesimal generator of an analytic semigroup on $L^{2}(\Omega)$. Its resolvent is compact.

Proof. The proof relies on the following inequality

$$
\left(\left(\lambda_{0} I-A\right) z, z\right)_{L^{2}(\Omega)} \geq \frac{\nu}{2}\|z\|_{H^{1}(\Omega)}^{2} \quad \forall z \in \mathcal{D}(A),
$$

with $\lambda_{0}>0$ large enough. See e.g. [38], or [31] where a similar estimate is established for the Oseen operator.

Notice that $\left(A^{*}, \mathcal{D}\left(A^{*}\right)\right)$ is defined by

$$
\mathcal{D}\left(A^{*}\right)=\left\{\phi \in H^{2}(\Omega) \mid \phi=0 \text { on } \Gamma_{d} \text { and } \nu \frac{\partial \phi}{\partial n}+w_{s} \phi=0 \text { on } \Gamma_{n}\right\},
$$

and

$$
A^{*} \phi=\nu \Delta \phi+\left(\partial_{1} \phi+\partial_{2} \phi\right) w_{s} \text { for all } \phi \in \mathcal{D}\left(A^{*}\right) .
$$

With the extrapolation method, the operator $A$ can be extended as an unbounded operator in $\left(\mathcal{D}\left(A^{*}\right)\right)^{\prime}$ with domain $\mathcal{D}\left(A ;\left(\mathcal{D}\left(A^{*}\right)\right)^{\prime}\right)=Z$.

We introduce the Dirichlet operator $D \in \mathcal{L}\left(L^{2}\left(\Gamma_{d}\right), L^{2}(\Omega)\right)$, by setting $D v=\xi$, where $\xi$ is the solution to the following elliptic equation

$$
\lambda_{0} \xi-\nu \Delta \xi+\left(\partial_{1} w_{s}+\partial_{2} w_{s}\right) \xi+\left(\partial_{1} \xi+\partial_{2} \xi\right) w_{s}=0 \quad \text { in } \Omega, \quad \nu \frac{\partial \xi}{\partial n}=0 \quad \text { on } \Gamma_{n}, \quad \xi=v \quad \text { on } \Gamma_{d},
$$

where $\lambda_{0}>0$ is chosen so that (2.3) is true. From elliptic regularity results, it follows that $D$ is also continuous from $L^{2}\left(\Gamma_{d}\right)$ into $H^{1 / 2-\varepsilon}(\Omega)=\mathcal{D}\left(\left(\lambda_{0} I-A\right)^{1 / 4-\varepsilon / 2}\right)$ for all $\varepsilon>0$. For the definition of fractional power of positive operators we refer to [5]. We define $B \in \mathcal{L}\left(U,\left(\mathcal{D}\left(A^{*}\right)\right)^{\prime}\right)$ by

$$
B u=\sum_{i=1}^{N_{c}} u_{i}\left(\lambda_{0}-A\right) D g_{i} \quad \text { for all } u=\left(u_{1}, \ldots, u_{N_{c}}\right)^{T} \in U=\mathbb{R}^{N_{c}} .
$$

The operator $B$ is well defined because $D \in \mathcal{L}\left(L^{2}\left(\Gamma_{d}\right), L^{2}(\Omega)\right)$ and $A \in \mathcal{L}\left(L^{2}(\Omega),\left(\mathcal{D}\left(A^{*}\right)\right)^{\prime}\right)$. We use here the extension of $A$ defined via the extrapolation method. Moreover since $D$ also belongs to 
$\mathcal{L}\left(L^{2}\left(\Gamma_{d}\right), \mathcal{D}\left(\left(\lambda_{0} I-A\right)^{1 / 4-\varepsilon / 2}\right)\right)$, it follows that $\left.B \in \mathcal{L}\left(U,\left(\mathcal{D}\left(\left(\lambda_{0} I-A\right)^{3 / 4+\varepsilon / 2}\right)\right)\right)^{\prime}\right)$. In particular $\left(\lambda_{0} I-\right.$ $A)^{-3 / 4-\varepsilon / 2} B \in \mathcal{L}\left(U, L^{2}(\Omega)\right)$. Thus

$$
\left(\lambda_{0} I-A\right)^{-\alpha} B \in \mathcal{L}(U, Z) \text { for all } 3 / 4<\alpha \leq 1 .
$$

When $g_{i} \in L^{2}\left(\Gamma_{d}\right)$ and $u_{i} \in L^{2}(0, \infty)$, the solutions to equation (1.7) may be defined by transposition [28], or they can also be defined as solutions to the evolution equation

$$
z^{\prime}=A z+B u, \quad z(0)=z_{0} .
$$

Proposition 2.2. The adjoint of $B \in \mathcal{L}\left(\mathbb{R}^{N_{c}},\left(\mathcal{D}\left(A^{*}\right)\right)^{\prime}\right)$ is the operator $B^{*} \in \mathcal{L}\left(\mathcal{D}\left(A^{*}\right), \mathbb{R}^{N_{c}}\right)$ defined by

$$
B^{*} \phi=\left(-\nu \int_{\Gamma_{c}} \frac{\partial \phi}{\partial n} g_{i}\right)_{1 \leq i \leq N_{c}} .
$$

Proof. The result follows from the Green formula.

Since $(A, \mathcal{D}(A))$ is the infinitesimal generator of an analytic semigroup and since its resolvent is compact, the eigenvalues of $A$ are isolated, with finite multiplicity, and for any $\omega>0$, there is only a finite number of eigenvalues with real part greater than $-\omega$. We denote by $\left(\lambda_{i}\right)_{1 \leq i \leq \infty}$ the eigenvalues of $A$ and we assume that the eigenvalues are numbered so that $\operatorname{Re} \lambda_{i} \geq \operatorname{Re} \lambda_{i+1}$ for all $i \in \mathbb{N}^{*}$. In order to look for a feedback control law providing a prescribed exponential decay rate $-\omega<0$, we choose $\omega$ such that $-\omega \notin \operatorname{Re} \sigma(A)$. Thus we have

$$
\ldots \leq \operatorname{Re} \lambda_{N_{\omega}+1}<-\omega<\operatorname{Re} \lambda_{N_{\omega}} \leq \ldots \leq \operatorname{Re} \lambda_{1}
$$

and $N_{\omega}$ is the number of eigenvalues with real part greater than $-\omega$. For each $1 \leq j \leq N_{\omega}$, we denote by $\left(\phi_{j}^{k}\right)_{1 \leq k \leq \ell_{j}}$ a basis of $\operatorname{Ker}\left(A^{*}-\lambda_{j}\right)$ (thus $\ell_{j}=\operatorname{dim}\left(\operatorname{Ker}\left(A^{*}-\lambda_{j}\right)\right)$ ). Since $A$ is not selfadjoint the eigenvalues $\lambda_{j}$ may be complex (there are pairs of complex conjugate eigenvalues), and the eigenfunctions $\phi_{j}^{k}$ of $A^{*}$ may be with complex values.

In order that the pair $(A+\omega I, B)$ is stabilizable, we choose the family $\left(g_{i}\right)_{1 \leq i \leq N_{c}}$ so that

$$
\text { for all } 1 \leq j \leq N_{\omega},
$$

$$
N_{c} \geq \ell_{j} \text { and the family }\left(\int_{\Gamma_{c}} \frac{\partial \phi_{j}^{k}}{\partial n} g_{1}, \ldots, \int_{\Gamma_{c}} \frac{\partial \phi_{j}^{k}}{\partial n} g_{N_{c}}\right)_{1 \leq k \leq \ell_{j}} \text { is of rank } \ell_{j} \text {. }
$$

In this setting, the functions $\frac{\partial \phi_{j}^{k}}{\partial n}$ take values in $\mathbb{C}$, while the functions $g_{i}$ take values in $\mathbb{R}$. It is always possible to choose the family $\left(g_{i}\right)_{1 \leq i \leq N_{c}}$ so that $\left(H_{4}\right)$ is satisfied (see e.g. [34]).

We need to introduce the following spaces

$$
H_{\Gamma_{d}}^{1}(\Omega)=\left\{z \in H^{1}(\Omega) \mid z=0 \text { on } \Gamma_{d}\right\} \quad \text { and } \quad H_{\Gamma_{d}}^{-1}(\Omega)=\left(H_{\Gamma_{d}}^{1}(\Omega)\right)^{\prime} .
$$

We have the following continuous and dense imbeddings

$$
H_{\Gamma_{d}}^{1}(\Omega) \hookrightarrow L^{2}(\Omega) \hookrightarrow H_{\Gamma_{d}}^{-1}(\Omega) .
$$

We have the following proposition.

Proposition 2.3. If assumption $\left(H_{4}\right)$ is fulfilled, then the pair $(A+\omega I, B)$ is stabilizable in $Z=L^{2}(\Omega)$ and in $H_{\Gamma_{d}}^{-1}(\Omega)$, with controls in $U$.

Proof. It is a consequence of the Hautus criterion, see [6,10] or [39], see also [33]. 


\subsection{Feedback control law}

In order to obtain the feedback stabilization of equation (2.6) with a given exponential decay rate $\omega>0$, we consider the equation

$$
z^{\prime}=(A+\omega I) z+B u, \quad z(0)=z_{0} .
$$

A way for finding a stabilizing feedback operator for equation (2.7) consists in looking for the solution to the following algebraic Riccati equation

$$
\begin{aligned}
& P \in \mathcal{L}(Z), P^{*}=P, P \geq 0, \quad(A+\omega I)-B R^{-1} B^{*} P \quad \text { is stable, } \\
& P(A+\omega I)+\left(A^{*}+\omega I\right) P-P B R^{-1} B^{*} P+Q=0,
\end{aligned}
$$

where $R \in \mathcal{L}(U), R=R^{*}>0, Q \in \mathcal{L}(Z), Q=Q^{*} \geq 0$. The operator $Q$ can be rewritten as $Q^{1 / 2} Q^{1 / 2}$. When the pair $(A+\omega I, B)$ is stabilizable and the pair $\left(A+\omega I, Q^{1 / 2}\right)$ is detectable, the equation

$$
P \in \mathcal{L}(Z), P^{*}=P, P \geq 0, \quad P(A+\omega I)+\left(A^{*}+\omega I\right) P-P B R^{-1} B^{*} P+Q=0,
$$

admits a unique solution. If the pair $\left(A+\omega I, Q^{1 / 2}\right)$ is not detectable, for example if $Q=0$, the uniqueness of solution to the above Riccati equation can be obtained by adding the condition that $(A+\omega I)-B R^{-1} B^{*} P$ is stable (see e.g. [23]). This is what is done in (2.8).

If $P$ is the solution to (2.8), then $K=-R^{-1} B^{*} P$ is a feedback gain stabilizing equation (2.7) in $Z$. Thus $A+\omega I+B K$, with domain $\mathcal{D}(A+\omega I+B K ; Z)=\{z \in Z \mid(A+B K) z \in Z\}$, is the generator of an analytic semigroup on $Z$, exponentially stable on $Z$. Let us notice that $\mathcal{D}\left((A+B K)^{*}\right)=\mathcal{D}\left(A^{*}\right)$ (see $\left.[1,2]\right)$. Thus, the operator $A+B K$ can be extended by extrapolation to $\left(\mathcal{D}\left((-A-B K)^{*}\right)\right)^{\prime}=\left(\mathcal{D}\left(A^{*}\right)\right)^{\prime}$ and to $H_{\Gamma_{d}}^{-1}(\Omega)=\left(\mathcal{D}\left(\lambda_{0} I-A^{*}\right)^{1 / 2}\right)^{\prime}=\left(\mathcal{D}\left(\left((-A-B K)^{*}\right)^{1 / 2}\right)\right)^{\prime}$. This is summarized in the following theorem.

Theorem 2.4. We assume that $\left(H_{4}\right)$ is satisfied. There exists an operator $K \in \mathcal{L}\left(H_{\Gamma_{d}}^{-1}(\Omega), U\right)$ such that the following properties are satisfied.

(i) $A+\omega I+B K$, with domain $\mathcal{D}(A+\omega I+B K ; Z)=\{z \in Z \mid(A+B K) z \in Z\}$, is the generator of an analytic semigroup on $Z$, exponentially stable on $Z$. Moreover

$$
\mathcal{D}(A+\omega I+B K ; Z)=\left\{z \in H^{2}(\Omega) \mid z=\sum_{i=1}^{N_{c}}(K z)_{i} g_{i} \quad \text { on } \Gamma_{d}, \frac{\partial z}{\partial n}=0 \quad \text { on } \Gamma_{n}\right\} .
$$

(ii) $A+\omega I+B K$, with domain $\mathcal{D}\left(A+\omega I+B K ; H_{\Gamma_{d}}^{-1}(\Omega)\right)=\left\{z \in Z \mid(A+B K) z \in H_{\Gamma_{d}}^{-1}(\Omega)\right\}$, is the generator of an analytic semigroup on $H_{\Gamma_{d}}^{-1}(\Omega)$, exponentially stable on $H_{\Gamma_{d}}^{-1}(\Omega)$.

Proof. The existence of an operator $K \in \mathcal{L}(Z, U)$ for which $(i)$ is satisfied is well known, see e.g. [26]. What is new in this statement is the existence of $K \in \mathcal{L}\left(H_{\Gamma_{d}}^{-1}(\Omega), U\right)$ for which (i) and (ii) are satisfied with the same operator $K$.

Let us notice that, due to $(2.1)$, we have $H_{\Gamma_{d}}^{-1}(\Omega)=\left[\left(\mathcal{D}\left(A^{*}\right)\right)^{\prime}, Z\right]_{1 / 2}$. The existence of a feedback $K \in$ $\mathcal{L}\left(H_{\Gamma_{d}}^{-1}(\Omega), U\right)$ for which (i) and (ii) are satisfied simultaneously is established in [32] for the Oseen equation. The adaptation to the linearized Burgers equation is straightforward. Next, other feedback operators satisfying the properties (i) and (ii) have been constructed in [34] by using control spaces of finite dimension. The same approach can be followed here. The identity $\mathcal{D}(A+\omega I+B K ; Z)=\left\{z \in H^{2}(\Omega) \mid z=K z \quad\right.$ on $\Gamma_{d}$, $\frac{\partial z}{\partial n}=$ 0 on $\left.\Gamma_{n}\right\}$ follows from elliptic regularity results (see e.g. $[18,19]$ ). In the particular case of a rectangle with either a Dirichlet boundary condition or a Neumann boundary condition on each side of the rectangle, the $H^{2}(\Omega)$-regularity can be also obtained by using symmetry arguments.

Corollary 2.5. We assume that $\left(H_{4}\right)$ is satisfied. Let $K \in \mathcal{L}\left(H_{\Gamma_{d}}^{-1}(\Omega), U\right)$ satisfy the conclusions of Theorem 2.4. The operator $A+B K$ is an isomorphism from $H_{\mathcal{F}}^{1}(\Omega)=\left\{z \in H^{1}(\Omega) \mid(A+B K) z \in H_{\Gamma_{d}}^{-1}(\Omega)\right\}$, equipped with the $H^{1}(\Omega)$ norm, into $H_{\Gamma_{d}}^{-1}(\Omega)$. Moreover $H_{\mathcal{F}}^{1}(\Omega)=[Z, \mathcal{D}(A+B K)]_{1 / 2}$.

Proof. This is an easy consequence of Theorem 2.4. 


\subsection{Filtering operator}

Let us recall that $H$ is the operator defined in (1.9). Let us study the detectability of the pair $(A+\omega I, H)$. For each $1 \leq j \leq N_{\omega}$, we denote by $\left(\psi_{j}^{k}\right)_{1 \leq k \leq \ell_{j}}$ a basis of $\operatorname{Ker}\left(A-\lambda_{j}\right)$. We assume that

for all $1 \leq j \leq N_{\omega}$,

$$
N_{o} \geq \ell_{j} \text { and the family }\left(\frac{1}{\left|\Gamma_{1}\right|} \int_{\Gamma_{1}} \psi_{j}^{k}, \ldots, \frac{1}{\left|\Gamma_{N_{o}}\right|} \int_{\Gamma_{N_{o}}} \psi_{j}^{k}\right)_{1 \leq k \leq \ell_{j}} \quad \text { is of rank } \ell_{j} \text {. }
$$

In the case when $w_{s} \equiv 0$, it could be proved, by using the explicit expression of the families $\left(\psi_{j}^{k}\right)_{1 \leq k \leq \ell_{j}}$, that the family of intervals $\left(\Gamma_{i}\right)_{1 \leq i \leq N_{o}}$ can be chosen so that $\left(H_{5}\right)$ is satisfied. We conjecture that this is still the case when $w_{s} \not \equiv 0$, but we do not investigate that issue since it is not our main objective. Numerical tests may also be used to check that assumption (see Sect. 5.3).

Proposition 2.6. If assumption $\left(H_{5}\right)$ is fulfilled, then the pair $(A+\omega I, H)$ is detectable in $Z$.

Proof. It is still a consequence of the Hautus criterion, see $[6,10,39]$.

It is clear that the operator $H$ obeys

$$
\|H z\|_{Y} \leq C\left\|\left.z\right|_{\Gamma_{n}}\right\|_{L^{2}\left(\Gamma_{n}\right)}
$$

Due to (2.9), we have

$$
H\left(\lambda_{0} I-A\right)^{-\beta} \in \mathcal{L}(Z, Y), \quad \text { for all } 1 / 4<\beta<1 .
$$

We look for $L \in \mathcal{L}(Y, Z)$ such that $A+\omega I+L H$, with domain $\mathcal{D}(A+\omega I+L H)=\{z \in Z \mid(A+L H) z \in$ $Z\}=\mathcal{D}(A)$, is the generator of an analytic semigroup on $Z$, exponentially stable on $Z$.

Under the above conditions, if $R_{\eta} \in \mathcal{L}(Y), R_{\eta}=R_{\eta}^{*}>0, Q_{\mu} \in \mathcal{L}(Z), Q_{\mu}=Q_{\mu}^{*} \geq 0$, the equation

$$
\begin{aligned}
& P_{e} \in \mathcal{L}(Z), P_{\mathrm{e}}^{*}=P_{e}, P_{e} \geq 0, \quad A-P_{e} H^{*} R_{\eta}^{-1} H \quad \text { is stable } \\
& (A+\omega I) P_{e}+P_{e}\left(A^{*}+\omega I\right)-P_{e} H^{*} R_{\eta}^{-1} H P_{e}+Q_{\mu}=0
\end{aligned}
$$

admits a unique solution (see e.g. [26]). We choose $L \in \mathcal{L}(Y, Z)$ as

$$
L=-P_{e} H^{*} R_{\eta}^{-1} \text {. }
$$

\subsection{The estimation equation}

In this section, we want to study the equation

$$
z_{e}^{\prime}=A z_{e}+L\left(H z_{e}-y_{o b s}\right)+B K z_{e}, \quad z_{e}(0)=z_{e, 0} .
$$

We already know that $A+\omega I+B K$, with domain $\mathcal{D}(A+\omega I+B K)=\{z \in Z \mid(A+B K) z \in Z\}$, is the infinitesimal generator of an analytic semigroup exponentially stable on $Z$.

In order to study equation (2.12), we need the additional condition

$$
L H(-A-B K)^{-\gamma} \text { is bounded from } Z \text { into } Z,
$$

for some $0 \leq \gamma<1$.

Proposition 2.7. Let us assume that $\left(H_{4}\right)$ and $\left(H_{5}\right)$ are satisfied, $K$ satisfies the conclusions of Theorem 2.4, and $L$ is defined by $(2.11)$. Then assumption $\left(H_{6}\right)$ is satisfied. 
Proof. We are going to prove $\left(H_{6}\right)$ for $\gamma=1 / 2$. It is sufficient to prove that $H(-A-B K)^{-1 / 2} \in \mathcal{L}(Z, Y)$. Due to a density argument, this is equivalent to showing that

$$
\left\|H(-A-B K)^{-1 / 2}(-A-B K)^{-1 / 2} f\right\|_{Y} \leq C\left\|(-A-B K)^{-1 / 2} f\right\|_{Z} \quad \forall f \in Z .
$$

Let us consider the elliptic equation

$$
(A+B K) z=f, \quad \text { with } f \in Z .
$$

This equation admits a unique solution defined by

$$
z=-\int_{0}^{\infty} \mathrm{e}^{\tau(A+B K)} f \mathrm{~d} \tau=-(-A-B K)^{-1} f .
$$

Let us notice that $(-A-B K)^{-1 / 2} f=-(-A-B K)^{1 / 2} z$. It is easy to prove that, when $f \in Z=L^{2}(\Omega)$, equation (2.13) is equivalent to the elliptic boundary value problem

$$
\begin{aligned}
& \nu \Delta z-\left(\partial_{1} w_{s}+\partial_{2} w_{s}\right) z-\left(\partial_{1} z+\partial_{2} z\right) w_{s}=f \quad \text { in } \Omega \\
& \nu \frac{\partial z}{\partial n}=0 \quad \text { on } \Gamma_{n}, \quad z=\sum_{i=1}^{N_{c}}(K z)_{i} g_{i} \quad \text { on } \Gamma_{d} .
\end{aligned}
$$

Since $A+B K$ is an isomorphism from $H_{\mathcal{F}}^{1}\left(\Omega\right.$ into $H_{\Gamma_{d}}^{-1}(\Omega)$ (see Cor. 2.5), we have

$$
c_{1}\|z\|_{H_{\mathcal{F}}^{1}(\Omega)} \leq\|f\|_{H_{\Gamma_{d}}^{-1}(\Omega)} \leq c_{2}\|z\|_{H_{\mathcal{F}}^{1}(\Omega)}, \quad \text { with } 0<c_{1}<c_{2},
$$

for all $f \in H_{\Gamma_{d}}^{-1}(\Omega)$. In particular, we have

$$
\left\|\left.z\right|_{\Gamma_{n}}\right\|_{L^{2}\left(\Gamma_{n}\right)} \leq C\|f\|_{H_{\Gamma_{d}}^{-1}(\Omega)}
$$

Knowing that $H_{\mathcal{F}}^{1}(\Omega)=[Z, \mathcal{D}(A+B K)]_{1 / 2}$, we have

$$
\|z\|_{H_{\mathcal{F}}^{1}(\Omega)} \leq C\left\|(-A-B K)^{1 / 2} z\right\|_{L^{2}(\Omega)} .
$$

Collecting these different results, we obtain

$$
\begin{aligned}
& \left\|H(-A-B K)^{-1} f\right\|_{Y} \leq C\left\|\left.z\right|_{\Gamma_{n}}\right\|_{L^{2}\left(\Gamma_{n}\right)} \leq C\|f\|_{H_{\Gamma_{d}}^{-1}(\Omega)} \leq C\|z\|_{H_{\mathcal{F}}^{1}(\Omega)} \\
& \leq C\left\|(-A-B K)^{1 / 2} z\right\|_{L^{2}(\Omega)}=C\left\|(-A-B K)^{-1 / 2} f\right\|_{L^{2}(\Omega)} .
\end{aligned}
$$

Which implies that $\left(H_{6}\right)$ is satisfied for $\gamma=1 / 2$.

Proposition 2.8. Let us assume that $\left(H_{4}\right)$ and $\left(H_{5}\right)$ are satisfied, $K$ satisfies the conclusions of Theorem 2.4, and $L$ is defined by (2.11). Then the operator $A+B K+L H$, with domain $\mathcal{D}(A+B K+L H)=\mathcal{D}(A+B K)$, generates an analytic semigroup on $Z$. If $y_{o b s} \in L^{2}(0, \infty ; Y)$ and $z_{e, 0} \in Z$, then the solution $z_{e}$ to equation $(2.12)$ belongs to $L_{\mathrm{loc}}^{2}\left([0, \infty) ; \mathcal{D}\left((-A-B K)^{1 / 2}\right)\right) \cap H_{\mathrm{loc}}^{1}\left([0, \infty) ; \mathcal{D}\left(\left(\lambda_{0} I-A^{*}\right)^{-1 / 2}\right)\right)$.

Proof. Since $\left(H_{6}\right)$ is satisfied, from ([30], Chap. 3, Cor. 2.4), it follows that $A+B K+L H$, with domain $\mathcal{D}(A+$ $B K+L H)=\mathcal{D}(A+B K)$ is also the infinitesimal generator of an analytic semigroup on $Z$. Thus equation (2.12) will be well posed for any $y_{\text {obs }} \in L^{2}(0, \infty ; Y)$. The existence of the solution in $L_{\text {loc }}^{2}\left([0, \infty) ; \mathcal{D}\left((-A-B K)^{1 / 2}\right)\right) \cap$ $H_{\mathrm{loc}}^{1}\left([0, \infty) ;\left(\mathcal{D}\left(\left(\lambda_{0} I-A^{*}-K^{*} B^{*}-H^{*} L^{*}\right)^{1 / 2}\right)\right)^{\prime}\right)$ follows from ([5], Chap. 3, Thm. 2.2). Once we know that $z_{e}$ belongs to $L_{\text {loc }}^{2}\left([0, \infty) ; \mathcal{D}\left((-A-B K)^{1 / 2}\right)\right)$, the term $L H z_{e}$ can be considered as a source term in equation $(2.12)$, and we can prove that $z_{e}$ also belongs to $L_{\mathrm{loc}}^{2}\left([0, \infty) ; \mathcal{D}\left((-A-B K)^{1 / 2}\right)\right) \cap H_{\mathrm{loc}}^{1}\left([0, \infty) ; \mathcal{D}\left(\left(\lambda_{0} I-A^{*}\right)^{-1 / 2}\right)\right)$. 


\subsection{Additional results}

Let us consider the system

$$
\begin{aligned}
& z^{\prime}=A z+B K z_{e}, \quad z(0)=z_{0}+\mu_{0}, \\
& z_{e}^{\prime}=(A+B K+L H) z_{e}-L H z, \quad z_{e}(0)=z_{0},
\end{aligned}
$$

and the associated system satisfied by $(z, e)$ where $e=z-z_{e}$ :

$$
\begin{aligned}
& z^{\prime}=(A+B K) z-B K e, \quad z(0)=z_{0}+\mu_{0}, \\
& e^{\prime}=(A+L H) e, \quad e(0)=\mu_{0} .
\end{aligned}
$$

The goal of this section is to analyze the above two systems and their interconnections.

Theorem 2.9. We assume that assumptions $\left(H_{4}\right)-\left(H_{5}\right)$ are satisfied, $K \in \mathcal{L}\left(H_{\Gamma_{d}}^{-1}(\Omega), U\right)$ fulfils the conclusion of Theorem 2.4, and $L \in \mathcal{L}(Y, Z)$ is defined by (2.11). Then, we have

$$
\left\|\mathrm{e}^{t(A+B K)}\right\|_{\mathcal{L}(Z)} \leq M_{\omega} \mathrm{e}^{-\omega t} \text { and }\left\|\mathrm{e}^{t(A+L H)}\right\|_{\mathcal{L}(Z)} \leq M_{\omega} \mathrm{e}^{-\omega t},
$$

for some $M_{\omega} \geq 1$. Moreover, the operator $\mathcal{A}_{e}$ defined in $Z \times Z$ by

$$
\begin{aligned}
\mathcal{D}\left(\mathcal{A}_{e}\right) & =\{(z, e) \in Z \times Z \mid A z+B K z-B K e \in Z,(A+L H) e \in Z\}, \\
\mathcal{A}_{e} & =\left(\begin{array}{cc}
A+B K & -B K \\
0 & A+L H
\end{array}\right),
\end{aligned}
$$

generates an analytic semigroup exponentially stable on $Z \times Z$.

Proof. When $K \in \mathcal{L}\left(H_{\Gamma_{d}}^{-1}(\Omega), U\right)$ fulfils the conclusion of Theorem 2.4 and $L \in \mathcal{L}(Y, Z)$ is defined by (2.11), it is well known that the semigroups $\left(\mathrm{e}^{t(A+B K)}\right)_{t>0}$ and $\left(\mathrm{e}^{t(A+B K)}\right)_{t>0}$ are analytic and satisfy (2.17). It is clear that $\left(\mathcal{A}_{e}, \mathcal{D}\left(\mathcal{A}_{e}\right)\right)$ generates an exponentially stable semigroup on $Z \times Z$, because $B K$ is bounded from $Z$ into $\left(\mathcal{D}\left(\lambda_{0} I-A^{*}\right)^{\alpha}\right)^{\prime}$ and $\left(\mathcal{D}\left(\left((-A-B K)^{*}\right)^{\alpha}\right)\right)^{\prime}=\left(\mathcal{D}\left(\lambda_{0} I-A^{*}\right)^{\alpha}\right)^{\prime}$ (indeed $\left.\mathcal{D}\left((A+B K)^{*}\right)=\mathcal{D}\left(A^{*}\right)\right)$. We can verify that the semigroup generated by $\mathcal{A}_{e}$ is analytic. We can use ([5], Part II, Chap. 3, Thm. 2.2) to obtain the existence of a unique solution to system (2.16).

From Theorem 2.9, it follows that the family of operators

$$
\left(\left(z_{0}+\mu_{0}, z_{0}\right) \mapsto\left(z(t), z_{e}(t)\right)\right)_{t \geq 0},
$$

where $z_{e}(t)=z(t)-e(t)$ and $(z, e)$ is the solution to (2.16) corresponding to the initial data $\left(z_{0}, e_{0}\right)=\left(z_{0}+\right.$ $\left.\mu_{0}, \mu_{0}\right)$, is the infinitesimal generator of an exponentially stable semigroup on $Z \times Z$. We set

$$
\mathcal{D}(\mathcal{A})=\left\{\left(z, z_{e}\right) \in Z \times Z \mid A z+B K z_{e} \in Z, z-z_{e} \in \mathcal{D}(A),-L H\left(z-z_{e}\right)+(A+B K) z_{e} \in Z\right\}
$$

and

$$
\mathcal{A}=\left(\begin{array}{cc}
A & B K \\
-L H & A+B K+L H
\end{array}\right) .
$$

Let us notice that $L H\left(z-z_{e}\right)$ is meaningful because $z-z_{e} \in \mathcal{D}(A)$, while $L H z$ and $L H z_{e}$ are not necessarily defined separately. However in the particular situation corresponding to the linearized Burgers equation studied here, if $A z+B K z_{e} \in Z$, then $z \in H^{2}(\Omega)$, thus $H z$ and $L H z$ are well defined and $H z_{e}$ and $L H z_{e}$ are also well defined. Thus in the particular case considered here, we can easily verify the results stated in the two following theorems. 
Theorem 2.10. Let us assume that hypotheses $\left(H_{4}\right)-\left(H_{5}\right)$ are satisfied, $K \in \mathcal{L}\left(H_{\Gamma_{d}}^{-1}(\Omega), U\right)$ fulfills the conclusion of Theorem 2.4, and $L \in \mathcal{L}(Y, Z)$ is defined by (2.11). Then $(z, e)$ belongs to $\mathcal{D}\left(\mathcal{A}_{e}\right)$ if and only if $\left(z, z_{e}\right)=(z, z-e)$ belongs to $\mathcal{D}(\mathcal{A})$.

Theorem 2.11. Let us assume that hypotheses $\left(H_{4}\right)-\left(H_{5}\right)$ are satisfied, $K \in \mathcal{L}\left(H_{\Gamma_{d}}^{-1}(\Omega), U\right)$ fulfills the conclusion of Theorem 2.4, and $L \in \mathcal{L}(Y, Z)$ is defined by (2.11). A pair $(z, e)$ is the solution to equation (2.16) if, and only if, $\left(z, z_{e}\right)=(z, z-e)$ is the solution to equation $(2.15)$. Moreover the operator $(\mathcal{A}, \mathcal{D}(\mathcal{A}))$ is the infinitesimal generator of an exponentially stable semigroup on $Z \times Z$.

\section{LOCAL STABILIZATION OF THE NONLINEAR SYSTEM}

In this section, we want to prove a local stabilization result for the system

$$
\begin{aligned}
& z^{\prime}=A z+B K z_{e}+F\left(w_{\zeta}+z\right)+\mu, \quad z(0)=z_{0}+\mu_{0}, \\
& z_{e}^{\prime}=A z_{e}+L\left(H z_{e}-y_{o b s}\right)+B K z_{e}, \quad z_{e}(0)=z_{0},
\end{aligned}
$$

with the observation

$$
y_{\text {obs }}(t)=H z(t)+\eta(t) .
$$

Let us recall that $F\left(w_{\zeta}+z\right)=\left(\partial_{1}\left(w_{\zeta}+z\right)+\partial_{2}\left(w_{\zeta}+z\right)\right)\left(w_{\zeta}+z\right)$.

Since we look for solutions satisfying a prescribed exponential decay rate $-\omega$, we make the following change of unknowns

$$
\widetilde{z}=\mathrm{e}^{\omega t} z, \quad \widetilde{z}_{e}=\mathrm{e}^{\omega t} z_{e}, \quad \widetilde{w}_{\zeta}=\mathrm{e}^{\omega t} w_{\zeta}, \quad \tilde{\mu}=\mathrm{e}^{\omega t} \mu,
$$

and we denote by $\widetilde{y}_{\text {obs }}$ the noisy observation of $\widetilde{z}$, that is

$$
\widetilde{y}_{o b s}(t)=H \widetilde{z}+\widetilde{\eta}(t), \quad \text { with } \widetilde{\eta}(t)=\mathrm{e}^{\omega t} \eta(t) .
$$

Thus equation (3.1) may be rewritten in the form

$$
\begin{aligned}
& \widetilde{z}^{\prime}=(A+\omega I) \widetilde{z}+B K \widetilde{z}_{e}+\mathrm{e}^{-\omega t} F\left(\widetilde{w}_{\zeta}+\widetilde{z}\right)+\widetilde{\mu}, \quad \widetilde{z}(0)=z_{0}+\mu_{0}, \\
& \widetilde{z}_{e}^{\prime}=(A+\omega I) \widetilde{z}_{e}+L\left(H \widetilde{z}_{e}-\widetilde{y}_{o b s}\right)+B K \widetilde{z}_{e}, \quad \widetilde{z}_{e}(0)=z_{0} .
\end{aligned}
$$

If we write the system satisfied by $\widetilde{z}$ and $\widetilde{e}=\widetilde{z}-\widetilde{z}_{e}$, we obtain

$$
\begin{aligned}
& \widetilde{z}^{\prime}=(A+\omega I) \widetilde{z}+B K \widetilde{z}-B K \widetilde{e}+\mathrm{e}^{-\omega t} F\left(\widetilde{w}_{\zeta}+\widetilde{z}\right)+\widetilde{\mu}, \quad \widetilde{z}(0)=z_{0}+\mu_{0}, \\
& \widetilde{e}^{\prime}=(A+\omega I) \widetilde{e}+L H \widetilde{e}-L H \widetilde{\eta}+\mathrm{e}^{-\omega t} F\left(\widetilde{w}_{\zeta}+\widetilde{z}\right)+\widetilde{\mu}, \quad \widetilde{e}(0)=z_{0} .
\end{aligned}
$$

Remark 3.1. We are going to assume that $\widetilde{\mu}$ belongs to $L^{2}\left(0, \infty ; H_{\Gamma_{d}}^{-1+\varepsilon}(\Omega)\right)$. Since $H_{\Gamma_{d}}^{-1}(\Omega)=\left(\mathcal{D}\left(\lambda_{0} I-\right.\right.$ $\left.\left.A^{*}\right)^{1 / 2}\right)^{\prime}=\left(\mathcal{D}\left(\left((-A-B K)^{*}\right)^{1 / 2}\right)\right)^{\prime}$ (see the proof of Thm. 2.4), with the reiteration theorem in interpolation, we can also show that $H_{\Gamma_{d}}^{-1+\varepsilon}(\Omega)=\left(\mathcal{D}\left(\lambda_{0} I-A^{*}\right)^{1 / 2-\varepsilon / 2}\right)^{\prime}=\left(\mathcal{D}\left(\left((-A-B K)^{*}\right)^{1 / 2-\varepsilon / 2}\right)\right)^{\prime}$. This result will be very helpful to study the first equation in system (3.4).

Remark 3.2. To study the second equation in system (3.4), we need an additional assumption on $A+L H$, see $\left(H_{7}\right)$ below, in order to take into account the nonlinear term $e^{-\omega t} F\left(\widetilde{w}_{\zeta}+\widetilde{z}\right)$ of the equation. This assumption can be easily verified when the measure oparator is defined by (1.9), see Proposition 3.3.

$\left(H_{7}\right)$ The following imbeddings are satisfied

$$
\mathcal{D}\left(\left((-A-L H)^{*}\right)^{1 / 2-\varepsilon / 2}\right) \hookrightarrow L^{1 / \varepsilon}(\Omega) \quad \text { and } \quad L^{1 /(1-\varepsilon)}(\Omega) \hookrightarrow\left(\mathcal{D}\left(\left((-A-L H)^{*}\right)^{1 / 2-\varepsilon / 2}\right)\right)^{\prime},
$$

for all $\varepsilon \in(0,1 / 2)$.

Let us now verify that assumption $\left(H_{7}\right)$ is satisfied by the operators $A$ and $H$ respectively defined by $(2.1)$ and (1.9). 
Proposition 3.3. We assume that $\left(H_{4}\right)$ and $H_{5}$ are satisfied, $A$ is defined by (2.1), $H$ is defined by (1.9) and $L$ is defined by (2.11). Then assumption $\left(H_{7}\right)$ is satisfied.

Proof.

Step 1. We first show that

$$
\|\phi\|_{H^{2-\tilde{\varepsilon}}(\Omega)} \leq C_{\tilde{\varepsilon}}\|\phi\|_{\mathcal{D}\left(A^{*}+H^{*} L^{*}\right)}, \quad \forall \phi \in \mathcal{D}\left(A^{*}+H^{*} L^{*}\right), \quad \text { and all } 0<\tilde{\varepsilon}<1 / 2 .
$$

The operator $L^{*}$ belongs to $\mathcal{L}\left(L^{2}(\Omega), \mathbb{R}^{N_{o}}\right)$. Let us set

$$
L^{*} \phi=\left(r_{1}^{\phi}, \ldots, r_{N_{o}}^{\phi}\right) \in \mathbb{R}^{N_{o}} .
$$

We have

$$
\left\|\left(r_{1}^{\phi}, \ldots, r_{N_{o}}^{\phi}\right)\right\|_{\mathbb{R}^{N_{o}}} \leq C\|\phi\|_{Z}
$$

Since $A^{*}+H^{*} L^{*}$ is an isomorphism from $\mathcal{D}\left(A^{*}+H^{*} L^{*}\right)$ into $Z$, we set $\|\phi\|_{\mathcal{D}\left(A^{*}+H^{*} L^{*}\right)}=\left\|\left(A^{*}+H^{*} L^{*}\right) \phi\right\|_{Z}$. The equation $\left(A^{*}+H^{*} L^{*}\right) \phi=f$ is equivalent to the system

$$
\begin{aligned}
\lambda_{0} \phi-\nu \Delta \phi-\left(\partial_{1} \phi+\partial_{2} \phi\right) w_{s} & =\lambda_{0} \phi-f \text { in } \Omega, \\
\nu \frac{\partial \phi}{\partial n}+w_{s} \phi & =\sum_{i=1}^{N_{o}} \frac{r_{i}^{\phi}}{\left|\Gamma_{i}\right|} \chi_{\Gamma_{i}} \text { on } \Gamma_{n}, \quad \phi=0 \text { on } \Gamma_{d}, \\
\left(r_{1}^{\phi}, \ldots, r_{N_{o}}^{\phi}\right) \in \mathbb{R}^{N_{o}} & =L^{*} \phi .
\end{aligned}
$$

Since $\sum_{i=1}^{N_{o}} \frac{r_{i}^{\phi}}{\Gamma_{i} \mid} \chi_{\Gamma_{i}}$ belongs to $H^{1 / 2-\tilde{\varepsilon}}\left(\Gamma_{n}\right)$ for all $\tilde{\varepsilon} \in(0,1 / 2)$, from elliptic regularity results it follows that

$$
\|\phi\|_{H^{2-\tilde{\varepsilon}}(\Omega)} \leq \tilde{C}_{\tilde{\varepsilon}}\left(\left\|\left(r_{1}^{\phi}, \ldots, r_{N_{o}}^{\phi}\right)\right\|_{\mathbb{R}^{N_{o}}}+\left\|\lambda_{0} \phi-f\right\|_{Z}\right) \leq C_{\tilde{\varepsilon}}\|f\|_{Z}=C_{\tilde{\varepsilon}}\|\phi\|_{\mathcal{D}\left(A^{*}+H^{*} L^{*}\right)},
$$

for all $\tilde{\varepsilon} \in(0,1 / 2)$ and all $\phi \in \mathcal{D}\left(A^{*}+H^{*} L^{*}\right)$.

Step 2. The identity mapping is linear and continuous from $\mathcal{D}\left(A^{*}+H^{*} L^{*}\right)$ into $H^{2-\tilde{\varepsilon}}(\Omega)$ and from $Z$ into $Z$. Thus it is also continuous from $\left[Z, \mathcal{D}\left(A^{*}+H^{*} L^{*}\right)\right]_{1 / 2-\varepsilon / 2}=\mathcal{D}\left(\left((-A-L H)^{*}\right)^{1 / 2-\varepsilon / 2}\right)$ into $\left[Z, H^{2-\tilde{\varepsilon}}(\Omega)\right]_{1 / 2-\varepsilon / 2}=H^{1-\varepsilon-\tilde{\varepsilon} / 2+\varepsilon \tilde{\varepsilon} / 2}(\Omega)$. Since $H^{1-\varepsilon-\tilde{\varepsilon} / 2+\varepsilon \tilde{\varepsilon} / 2}(\Omega) \hookrightarrow L^{1 / \varepsilon}(\Omega)$ for $\tilde{\varepsilon}>0$ small enough, we have $\mathcal{D}\left(\left((-A-L H)^{*}\right)^{1 / 2-\varepsilon / 2}\right) \hookrightarrow H^{1-\varepsilon-\tilde{\varepsilon} / 2+\varepsilon \tilde{\varepsilon} / 2}(\Omega) \hookrightarrow L^{1 / \varepsilon}(\Omega)$, with continuous imbeddings. Since $\mathcal{D}\left(\left((-A-L H)^{*}\right)^{1 / 2-\varepsilon / 2}\right)$ is dense in $L^{1 / \varepsilon}(\Omega)$, we also have $L^{1 /(1-\varepsilon)}(\Omega) \hookrightarrow\left(\mathcal{D}\left(\left((-A-L H)^{*}\right)^{1 / 2-\varepsilon / 2}\right)\right)^{\prime}$. The proof is complete.

We rewrite system (3.4) in the form

$$
\begin{aligned}
& \left(\begin{array}{l}
\widetilde{z} \\
\widetilde{e}
\end{array}\right)^{\prime}=\left(\begin{array}{cc}
A+\omega I+B K & -B K \\
0 & A+\omega I+L H
\end{array}\right)\left(\begin{array}{l}
\widetilde{z} \\
\widetilde{e}
\end{array}\right)+\left(\begin{array}{c}
\mathrm{e}^{-\omega t} F\left(\widetilde{w}_{\zeta}+\widetilde{z}\right)+\widetilde{\mu} \\
\mathrm{e}^{-\omega t} F\left(\widetilde{w}_{\zeta}+\widetilde{z}\right)+\widetilde{\mu}-L H \widetilde{\eta}
\end{array}\right), \\
& \left(\begin{array}{c}
\widetilde{z} \\
\widetilde{e}
\end{array}\right)(0)=\left(\begin{array}{c}
z_{0}+\mu_{0} \\
z_{0}
\end{array}\right) .
\end{aligned}
$$

Before stating our local stabilization result, let us recall the following definition (see e.g. [29])

$$
H^{1+\varepsilon, 1 / 2+\varepsilon / 2}(\Omega \times(0, \infty))=L^{2}\left(0, \infty ; H^{1+\varepsilon}(\Omega)\right) \cap H^{1 / 2+\varepsilon / 2}\left(0, \infty ; L^{2}(\Omega)\right) .
$$

Theorem 3.4. We assume that assumptions $\left(H_{4}\right)$ and $\left(H_{5}\right)$ are satisfied. Let $\varepsilon \in(0,1 / 2)$ be given fixed. There exists a constant $C_{0}>0$ and a nondecreasing function $\theta$ from $\mathbb{R}^{+}$into itself, such that, if $C \in\left(0, C_{0}\right)$, $z_{0}$ and $\mu_{0}$ belong to $H^{\varepsilon}(\Omega), \widetilde{w}_{\zeta}$ belongs to $H^{1+\varepsilon, 1 / 2+\varepsilon / 2}(\Omega \times(0, \infty))$, and

$$
\left\|z_{0}\right\|_{H^{\varepsilon}(\Omega)}+\left\|\mu_{0}\right\|_{H^{\varepsilon}(\Omega)}+\left\|\mathrm{e}^{\omega \cdot} \cdot \mu\right\|_{L^{2}\left(0, \infty ; H_{\Gamma_{d}}^{-1+\varepsilon}(\Omega)\right)}+\left\|\mathrm{e}^{\omega \cdot} w_{\zeta}\right\|_{H^{1+\varepsilon, 1 / 2+\varepsilon / 2}(\Omega \times(0, \infty))}+\left\|\mathrm{e}^{\omega \cdot} \eta\right\|_{L^{2}(0, \infty ; Y)} \leq \theta(C),
$$


then, the system (3.4) admits a unique solution in the space

$$
\left\{(\widetilde{z}, \widetilde{e}) \in\left(H^{1+\varepsilon, 1 / 2+\varepsilon / 2}(\Omega \times(0, \infty))\right)^{2} \mid\|(\widetilde{z}, \widetilde{e})\|_{\left(H^{1+\varepsilon, 1 / 2+\varepsilon / 2}(\Omega \times(0, \infty))\right)^{2}} \leq C\right\} .
$$

In particular the solution to system (3.1) obeys

$$
\|z(t)\|_{H^{\varepsilon}(\Omega)}+\left\|z(t)-z_{e}(t)\right\|_{H^{\varepsilon}(\Omega)} \leq C \mathrm{e}^{-\omega t} .
$$

Proof. The proof is based on the Banach fixed point Theorem. Let $\xi$ belong to $H^{1+\varepsilon, 1 / 2+\varepsilon / 2}(\Omega \times(0, \infty))$. We assume that $\widetilde{w}_{\zeta}$ belongs to $H^{1+\varepsilon, 1 / 2+\varepsilon / 2}(\Omega \times(0, \infty))$. We want to estimate $F\left(\widetilde{w}_{\zeta}+\xi\right)=\left(\partial_{1}+\partial_{2}\right)\left(\widetilde{w}_{\zeta}+\xi\right)\left(\widetilde{w}_{\zeta}+\xi\right)$ in $L^{2}\left(0, \infty ; H_{\Gamma_{d}}^{-1+\varepsilon}(\Omega)\right)$. We have

$$
\begin{aligned}
& \left\|\partial_{1}\left(\widetilde{w}_{\zeta}+\xi\right)\left(\widetilde{w}_{\zeta}+\xi\right)\right\|_{L^{2}\left(0, \infty ; H_{\Gamma_{d}}^{-1+\varepsilon}(\Omega)\right)} \leq C\left\|\partial_{1}\left(\widetilde{w}_{\zeta}+\xi\right)\left(\widetilde{w}_{\zeta}+\xi\right)\right\|_{L^{2}\left(0, \infty ; L^{2 /(2-\varepsilon)}(\Omega)\right)} \\
& \leq C\left\|\partial_{1}\left(\widetilde{w}_{\zeta}+\xi\right)\left(\widetilde{w}_{\zeta}+\xi\right)\right\|_{L^{2}\left(0, \infty ; L^{1 /(1-\varepsilon)}(\Omega)\right)}\left\|\widetilde{w}_{\zeta}+\xi\right\|_{L^{\infty}\left(0, \infty ; L^{2 /(1-\varepsilon)}(\Omega)\right)} \\
& \leq C\left\|\partial_{1}\left(\widetilde{w}_{\zeta}+\xi\right)\right\|_{L^{2}\left(0, \infty ; L^{2 /(1-\varepsilon)}(\Omega)\right)}\|\|_{L^{2}\left(0, \infty ; H^{\varepsilon}(\Omega)\right)}\left\|\widetilde{w}_{\zeta}+\xi\right\|_{L^{\infty}\left(0, \infty ; H^{\varepsilon}(\Omega)\right)} \\
& \leq C\left\|\partial_{1}\left(\widetilde{w}_{\zeta}+\xi\right)\right\|_{L^{2}} . \xi \widetilde{w}_{\zeta}+\xi \|_{H^{1+\varepsilon, 1 / 2+\varepsilon / 2}(\Omega \times(0, \infty))} .
\end{aligned}
$$

The first inequality follows from the imbeddings $H_{\Gamma_{d}}^{1-\varepsilon}(\Omega) \hookrightarrow L^{2 / \varepsilon}(\Omega)$ and $L^{2 /(2-\varepsilon)}(\Omega) \hookrightarrow H_{\Gamma_{d}}^{-1+\varepsilon}(\Omega)$, the fourth one follows from the imbedding $H^{\varepsilon}(\Omega) \hookrightarrow L^{2 /(1-\varepsilon)}(\Omega)$, and the last one from the imbedding $H^{1+\varepsilon, 1 / 2+\varepsilon / 2}(\Omega \times(0, \infty)) \hookrightarrow L^{\infty}\left(0, \infty ; H^{\varepsilon}(\Omega)\right)$ (see Lem. 3.5). Thus $\mathrm{e}^{-\omega t} F\left(\widetilde{w}_{\zeta}+\xi\right)$ belongs to $L^{2}\left(0, \infty ; H_{\Gamma_{d}}^{-1+\varepsilon}(\Omega)\right)$. It also belongs to $L^{2}\left(0, \infty ; L^{1 /(1-\varepsilon)}(\Omega)\right)$. This follows from the above inequality by omitting the first line.

Let us denote by $\left(z_{\xi}, e_{\xi}\right)$ the solution to the system

$$
\begin{aligned}
& z_{\xi}^{\prime}=(A+\omega I) z_{\xi}+B K z_{\xi}-B K e_{\xi}+\mathrm{e}^{-\omega t} F\left(\widetilde{w}_{\zeta}+\xi\right)+\widetilde{\mu}, \quad z_{\xi}(0)=z_{0}+\mu_{0}, \\
& e_{\xi}^{\prime}=(A+\omega I) e_{\xi}+L H e_{\xi}-L H \widetilde{\eta}+\mathrm{e}^{-\omega t} F\left(\widetilde{w}_{\zeta}+\xi\right)+\widetilde{\mu}, \quad e_{\xi}(0)=z_{0} .
\end{aligned}
$$

Since $H_{\Gamma_{d}}^{-1+\varepsilon}(\Omega) \hookrightarrow\left(\mathcal{D}\left(\left((-A-B K)^{*}\right)^{1 / 2}\right)\right)^{\prime}$ and $L^{1 /(1-\varepsilon)}(\Omega) \hookrightarrow\left(\mathcal{D}\left(\left((-A-L H)^{*}\right)^{1 / 2-\varepsilon / 2}\right)\right)^{\prime}$, we have the estimate (see [5], Chap. 3, p. 165)

$$
\begin{aligned}
\left\|\left(z_{\xi}, e_{\xi}\right)\right\|_{\left(H^{1+\varepsilon, 1 / 2+\varepsilon / 2}(\Omega \times(0, \infty))\right)^{2}} \leq & C_{1}\left(\left\|\mathrm{e}^{-\omega t} F\left(\widetilde{w}_{\zeta}+\xi\right)\right\|_{L^{2}\left(0, \infty ; H_{\Gamma_{d}}^{-1+\varepsilon}(\Omega)\right)}\right. \\
& +\left\|\mathrm{e}^{-\omega t} F\left(\widetilde{w}_{\zeta}+\xi\right)\right\|_{L^{2}\left(0, \infty ; L^{1 /(1-\varepsilon)}(\Omega)\right)}+\|\widetilde{\mu}\|_{L^{2}\left(0, \infty ; H_{\Gamma_{d}}^{-1+\varepsilon}(\Omega)\right)} \\
& \left.+\|\widetilde{\eta}\|_{L^{2}(0, \infty ; Y)}+\left\|z_{0}\right\|_{H^{\varepsilon}(\Omega)}+\left\|\mu_{0}\right\|_{H^{\varepsilon}(\Omega)}\right) \\
\leq & C_{1}\left(\left\|\widetilde{w}_{\zeta}+\xi\right\|_{H^{1+\varepsilon, 1 / 2+\varepsilon / 2}(\Omega \times(0, \infty))}^{2}+\|\widetilde{\mu}\|_{L^{2}\left(0, \infty ; H_{\Gamma_{d}}^{-1+\varepsilon}(\Omega)\right)}+\|\widetilde{\eta}\|_{L^{2}(0, \infty ; Y)}\right. \\
& \left.+\left\|z_{0}\right\|_{H^{\varepsilon}(\Omega)}+\left\|\mu_{0}\right\|_{H^{\varepsilon}(\Omega)}\right) .
\end{aligned}
$$

As in [38], we can find $C_{0}>0$ and $\theta$ such that the nonlinear mapping

$$
\xi \longmapsto\left(z_{\xi}, e_{\xi}\right)
$$

is a mapping from

$$
V_{C}=\left\{(\widetilde{z}, \widetilde{e}) \in\left(H^{1+\varepsilon, 1 / 2+\varepsilon / 2}(\Omega \times(0, \infty))\right)^{2} \mid\|(\widetilde{z}, \widetilde{e})\|_{\left(H^{1+\varepsilon, 1 / 2+\varepsilon / 2}(\Omega \times(0, \infty))\right)^{2}} \leq C\right\},
$$

into itself, if $0<C \leq C_{0}$, and provided that

$$
\left\|z_{0}\right\|_{H^{\varepsilon}(\Omega)}+\left\|\mu_{0}\right\|_{H^{\varepsilon}(\Omega)}+\left\|\mathrm{e}^{\omega \cdot} \mu\right\|_{L^{2}\left(0, \infty ; H_{\Gamma_{d}}^{-1+\varepsilon}(\Omega)\right)}+\left\|\mathrm{e}^{\omega} \cdot w_{\zeta}\right\|_{H^{1+\varepsilon, 1 / 2+\varepsilon / 2}(\Omega \times(0, \infty))}+\left\|\mathrm{e}^{\omega \cdot} \eta\right\|_{L^{2}(0, \infty ; Y)} \leq \theta(C) .
$$


Next, we can show that it is also a contraction for $C_{0}$ suitably chosen. Thus system (3.4) admits a unique solution in $V_{C}$. From Theorem 2.11, it follows that (3.3) admits a unique solution in the metric space

$$
\left\{\left(\widetilde{z}, \widetilde{z}_{e}\right) \in\left(H^{1+\varepsilon, 1 / 2+\varepsilon / 2}(\Omega \times(0, \infty))\right)^{2} \mid\left\|\left(\widetilde{z}, \widetilde{z}-\widetilde{z}_{e}\right)\right\|_{\left(H^{1+\varepsilon, 1 / 2+\varepsilon / 2}(\Omega \times(0, \infty))\right)^{2}} \leq C\right\},
$$

equipped with the distance corresponding to the norm in $\left(H^{1+\varepsilon, 1 / 2+\varepsilon / 2}(\Omega \times(0, \infty))\right)^{2}$. The existence of a unique solution to system (3.1) follows from the fact that $\left(\widetilde{z}, \widetilde{z}_{e}\right)$ is a solution to system (3.3) if and only if $\left(z, z_{e}\right)=\mathrm{e}^{-\omega t}\left(\widetilde{z}, \widetilde{z}_{e}\right)$ is a solution to system (3.3). The proof is complete.

Lemma 3.5. We have the following continuous imbedding

$$
H^{1+\varepsilon, 1 / 2+\varepsilon / 2}(\Omega \times(0, \infty)) \hookrightarrow L^{\infty}\left(0, \infty ; H^{\varepsilon}(\Omega)\right) .
$$

Proof. This lemma is a direct consequence of a trace theorem by Grisvard ([17], Lem. 4.1). Let us explain why. Let $z$ belong to $H^{1+\varepsilon, 1 / 2+\varepsilon / 2}(\Omega \times(0, \infty))$. We first extend $z$ as a function $\widehat{z}$ belonging to $H^{1+\varepsilon, 1 / 2+\varepsilon / 2}(\Omega \times \mathbb{R})$ by setting $\widehat{z}(\cdot,-t)=z(\cdot, t)$ for $t>0$. It is clear that the mapping $z \longmapsto \widehat{z}$ is linear and continuous from $H^{1+\varepsilon, 1 / 2+\varepsilon / 2}(\Omega \times(0, \infty))$ into $H^{1+\varepsilon, 1 / 2+\varepsilon / 2}(\Omega \times \mathbb{R})$. To prove the lemma, we have to show that

$$
H^{1+\varepsilon, 1 / 2+\varepsilon / 2}(\Omega \times \mathbb{R}) \hookrightarrow L^{\infty}\left(\mathbb{R} ; H^{\varepsilon}(\Omega)\right) .
$$

For that, it is sufficient to prove that there exists $C>0$ such that

$$
\|z(t)\|_{H^{\varepsilon}(\Omega)} \leq C\|z\|_{H^{1+\varepsilon, 1 / 2+\varepsilon / 2}(\Omega \times \mathbb{R})}, \quad \forall t \in \mathbb{R} .
$$

If $\tau_{t_{0}}$ denotes the translation operator, i.e. the operator defined by

$$
\left(\tau_{t_{0}} z\right)(t)=z\left(t-t_{0}\right),
$$

we know that

$$
\left\|\tau_{t_{0}} z\right\|_{H^{1+\varepsilon, 1 / 2+\varepsilon / 2}(\Omega \times \mathbb{R})}=\|z\|_{H^{1+\varepsilon, 1 / 2+\varepsilon / 2}(\Omega \times \mathbb{R})}, \quad \forall t_{0} \in \mathbb{R} .
$$

Therefore to prove (3.8), it is sufficient to prove it for $t=0$. Let us denote by $E$ a linear continuous extension operator from $H^{1+\varepsilon}(\Omega)$ to $H^{1+\varepsilon}\left(\mathbb{R}^{2}\right)$, which is also continuous from $L^{2}(\Omega)$ to $L^{2}\left(\mathbb{R}^{2}\right)$. Thus $E$ can be also considered as a linear continuous extension operator from $H^{1+\varepsilon, 1 / 2+\varepsilon / 2}(\Omega \times \mathbb{R})$ to $H^{1+\varepsilon, 1 / 2+\varepsilon / 2}\left(\mathbb{R}^{2} \times \mathbb{R}\right)$. From Lemma 4.1 of [17], it follows that

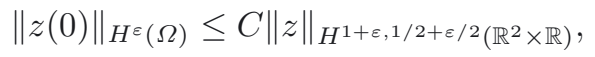

for all $z \in H^{1+\varepsilon, 1 / 2+\varepsilon / 2}\left(\mathbb{R}^{2} \times \mathbb{R}\right)$, and therefore

$$
\|z(0)\|_{H^{\varepsilon}(\Omega)} \leq C\|E z\|_{H^{1+\varepsilon, 1 / 2+\varepsilon / 2}\left(\mathbb{R}^{2} \times \mathbb{R}\right)} \leq C\|z\|_{H^{1+\varepsilon, 1 / 2+\varepsilon / 2}(\Omega \times \mathbb{R})},
$$

for all $z \in H^{1+\varepsilon, 1 / 2+\varepsilon / 2}(\Omega \times \mathbb{R})$. The proof of the lemma is complete.

\section{Approximate finite dimensional model}

\subsection{The model}

For the numerical simulations, we choose $L_{1}=L_{2}=1, \Omega=(0,1) \times(0,1)$, and we have $\Gamma_{d}=[0,1] \times\{0\}$ and $\Gamma_{n}=\Gamma \backslash \Gamma_{d}$. We choose a control space of dimension $1, U=\mathbb{R}$, with $g_{1}(x)=\sin \left(\pi x_{1}\right)$ in (1.1). The perturbation space is also choosen of dimension 1 in (1.1), with

$$
h_{1}(x)=\left\{\begin{array}{l}
\cos \left(\left(1-x_{2}\right) \frac{\pi}{2}\right) \quad \text { on } \Gamma_{n, \zeta}=\{0\} \times(0,1), \\
0 \quad \text { on } \Gamma_{n, 0}=\Gamma_{n} \backslash \Gamma_{n, \zeta},
\end{array}\right.
$$

see Figure 1. We choose

$$
w_{s}\left(x_{1}, x_{2}\right)=a x_{2}+b
$$




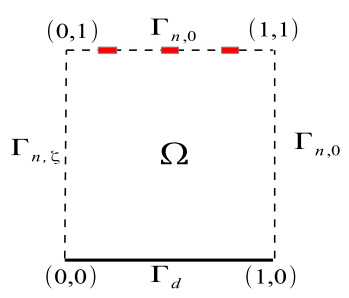

FiguRE 1. Domain and boundary conditions.

with $a=-0.068$ and $b=0.0365$ (see Sect. 5.2 where we explain this choice). This choice corresponds to $f_{s}=a\left(a x_{2}+b\right), g_{s}=b$ on $\Gamma_{d}$. The controlled system satisfied by $v=w-w_{s}$ is

$$
\begin{aligned}
& \frac{\partial v}{\partial t}=\nu \Delta v-\left(\partial_{1} v+\partial_{2} v\right) w_{s}-\left(\partial_{1} w_{s}+\partial_{2} w_{s}\right) v-\left(\partial_{1} v+\partial_{2} v\right) v \quad \text { in } \Omega \times(0, \infty), \\
& \nu \frac{\partial v}{\partial n}=h_{1}(x) \zeta(t) \quad \text { on } \Gamma_{n} \times(0, \infty), \quad v(x, t)=g_{1}(x) u(t) \quad \text { on } \Gamma_{d} \times(0, \infty), \\
& v(0)=v_{0}+\mu_{0} \quad \text { in } \Omega .
\end{aligned}
$$

As explained in Section 2, the above equation can be written in the form

$$
v^{\prime}=A v+B u+B_{d} \zeta+F(v), \quad v(0)=v_{0}+\mu_{0},
$$

with

$$
B u=u\left(\lambda_{0} I-A\right) D g_{1}, \quad B_{d} \zeta=\zeta\left(\lambda_{0} I-A\right) N h_{1}, \quad F(v)=\left(\partial_{1} v+\partial_{2} v\right) v,
$$

and where $\zeta\left(\lambda_{0} I-A\right) N h_{1}=w_{\zeta}$ is the solution to equation (1.5) when $N_{d}=1$. The measurements are of the form (1.9) and are precisely defined by

$$
y_{o b s}(t)=H v(t)+\eta(t),
$$

with

$$
\begin{aligned}
y_{o b s}(t) & =\left(y_{o b s, 1}(t), y_{o b s, 2}(t), y_{o b s, 3}(t)\right), \quad \eta(t)=\left(\eta_{1}(t), \eta_{2}(t), \eta_{3}(t)\right) \\
H v(t) & =\left(\frac{1}{0.05} \int_{0.2}^{0.25} v\left(x_{1}, 1, t\right), \frac{1}{0.05} \int_{0.5}^{0.55} v\left(x_{1}, 1, t\right), \frac{1}{0.05} \int_{0.8}^{0.85} v\left(x_{1}, 1, t\right)\right),
\end{aligned}
$$

and $\eta$ represents a measurement error. The sensor locations are drawn in red in Figure 1.

If we set $v=w_{\zeta}+z$, where $w_{\zeta}$ is the solution to (1.5) with $N_{d}=1$ and $h_{1}$ defined as above, the equation satisfied by $z$ is

$$
\begin{aligned}
\frac{\partial z}{\partial t}= & \nu \Delta z-\left(\partial_{1} z+\partial_{2} z\right) w_{s}-\left(\partial_{1} w_{s}+\partial_{2} w_{s}\right) z, \\
& -\left(\partial_{1}\left(w_{\zeta}+z\right)+\partial_{2}\left(w_{\zeta}+z\right)\right)\left(w_{\zeta}+z\right)+\mu \quad \text { in } \Omega \times(0, \infty), \\
\nu \frac{\partial z}{\partial n}= & 0 \quad \text { on } \Gamma_{n} \times(0, \infty), \quad z(x, t)=g_{1}(x) u(t) \quad \text { on } \Gamma_{d} \times(0, \infty), \\
z(0)= & v_{0}-w_{\zeta}(0)+\mu_{0} \quad \text { in } \Omega .
\end{aligned}
$$

with $\mu=-\frac{\partial w_{\zeta}}{\partial t}-\lambda_{0} w_{\zeta}$. We can also choose a function $w_{\zeta}$ which is not the solution to (1.5), for example

$$
w_{\zeta}\left(x_{1}, x_{2}, t\right)=\zeta(t) \frac{\nu}{2}\left(1-x_{1}\right)^{2} \cos \left(\frac{\pi}{2}\left(1-x_{2}\right)\right),
$$

and in that case, we have to choose $\mu=-\frac{\partial w_{\zeta}}{\partial t}+\nu \Delta w_{\zeta}-\left(\partial_{1} w_{s}+\partial_{2} w_{s}\right) w_{\zeta}-\left(\partial_{1} w_{\zeta}+\partial_{2} w_{\zeta}\right) w_{s}$. 
Thus we have a dynamical system of the form

$$
z^{\prime}=A z+B u+F\left(w_{\zeta}+z\right)+\mu, \quad z(0)=z_{0}+\mu_{0},
$$

as the one introduced in $(3.3)_{1}$. If we assume that $\zeta \in H^{1}(0, \infty)$, then $\mu$ belongs to $L^{2}\left(0, \infty ; L^{2}(\Omega)\right)$ and $w_{\zeta}$ belongs to $H^{1}\left(0, \infty ; H^{2}(\Omega)\right)$.

\subsection{Finite dimensional system}

To compute numerically the feedback gains and the solution of the compensator, we use a finite element method with $P_{1}$ basis functions on a structured triangular mesh [15]. The mesh size $h$ is equal to $1 / n$, which corresponds to a subdivision of each side of $\Omega$ into $n$ segments. We denote by $\mathcal{T}_{h}$ the corresponding partition of $\Omega$ into triangles. We introduce the two following finite dimensional spaces

$$
V_{h}:=\left\{\psi \in C^{0}(\bar{\Omega})|\psi|_{K} \in P_{1}, \forall K \in \mathcal{T}_{h}\right\},
$$

and

$$
V_{0 h}:=\left\{\psi \in C^{0}(\bar{\Omega})|\psi|_{K} \in P_{1}, \forall K \in \mathcal{T}_{h}, \psi=0 \text { on } \Gamma_{d}\right\} .
$$

We have $N:=\operatorname{dim}\left(V_{0 h}\right)=n(n-1)$ and $\operatorname{dim}\left(V_{h}\right)=n^{2}$. We denote by $\left(\varphi_{j}\right)_{j \in \mathcal{N}}$ the $n^{2}$ basis functions of $V_{h}$, where $\mathcal{N}$ is the set of nodes of the triangulation $\mathcal{T}_{h}$. We look for an approximate solution $v_{h} \in H^{1}\left(0, \infty ; V_{h}\right)$ of equation (4.1) of the form

$$
v_{h}(x, t):=\sum_{j \in \mathcal{N}_{\Omega \cup \Gamma_{n}}} v_{j}(t) \varphi_{j}(x)+\sum_{j \in \mathcal{N}_{\Gamma_{c}}} \sin \left(\pi a_{j}\right) \varphi_{j}(x) u(t),
$$

where $\mathcal{N}_{\Omega \cup \Gamma_{n}}$ is the set of nodes of $\mathcal{T}_{h}$ belonging to $\Omega \cup \Gamma_{n}, \mathcal{N}_{\Gamma_{c}}$ is the set of nodes of $\mathcal{T}_{h}$ belonging to $\Gamma_{c}$. Thus the discrete evolution equation associated with (4.1) is

Find $v_{h} \in H^{1}\left(0, \infty ; v_{h}\right)$ of the form (4.4) such that for all $\varphi \in V_{0 h}$,

$$
\begin{aligned}
\frac{\mathrm{d}}{\mathrm{d} t} \int_{\Omega} v_{h}(t) \varphi= & -\nu \int_{\Omega} \nabla v_{h} \cdot \nabla \varphi-\int_{\Omega}\left(\left(\partial_{x_{1}} w_{s, h}+\partial_{x_{2}} w_{s, h}\right) v_{h}+\left(\partial_{x_{1}} v_{h}+\partial_{x_{2}} v_{h}\right) w_{s, h}\right) \varphi \\
& -\int_{\Omega}\left(v_{h} \frac{\partial v_{h}}{\partial x_{1}}+v_{h} \frac{\partial v_{h}}{\partial x_{2}}\right) \varphi+\left(\int_{0}^{1} \cos \left(\frac{\pi}{2}\left(1-x_{2}\right)\right) \varphi\left(0, x_{2}\right) \mathrm{d} x_{2}\right) \zeta(t) \\
v_{h}(0)= & v_{0, h},
\end{aligned}
$$

where $v_{0, h}$ and $w_{s, h}$ are the finite element approximations of $v_{0}$ and $w_{s}$. By substituting $v_{h}$ defined by (4.4) in (4.5), we obtain one term involving the time derivative of the control $u$ :

$$
\left(\sum_{j \in \mathcal{N}_{\Gamma_{c}}} \sin \left(\pi a_{j}\right) \int_{\Omega} \varphi_{j} \varphi\right) u^{\prime}(t) .
$$

Hence the corresponding system of ordinary differential equations is not written in the form (1.8). To overcome this difficulty, we use a mass lumping method that is well known to compute, with high order elements, the solution of the wave equation [21] or parabolic equations [9]. For the space $P_{1}$, this method consists in calculating the integral on each triangle with a trapezoidal formula:

$$
\int_{K} \psi \approx \frac{|K|}{3}\left(\psi\left(a_{1}\right)+\psi\left(a_{2}\right)+\psi\left(a_{3}\right)\right)
$$

where the nodes $a_{1}, a_{2}, a_{3}$ are the vertices of triangle $K$. It follows that the mass matrix becomes diagonal since

$$
\left(\varphi_{i}, \varphi_{j}\right)_{h}=\delta_{i j} \frac{|K|}{3}
$$


where $(\cdot, \cdot)_{h}$ is an approximation of the $L_{2}(\Omega)$ inner product. Using this integration formula, we approximate the integral in (4.6) by zero, because $\varphi \in v_{0 h}$ and $j \in \mathcal{N}_{\Gamma_{c}}$. Thus, system (4.5) is of the form

$$
\mathbf{E v}^{\prime}=\mathbf{A} \mathbf{v}+\mathbf{B} u+\mathbf{B}_{d} \zeta+\mathbf{F}(\mathbf{v}), \quad \mathbf{v}(0)=\mathbf{v}_{0},
$$

where $\mathbf{A} \in \mathbb{R}^{N \times N}$ is the stiffness matrix, $\mathbf{E} \in \mathbb{R}^{N \times N}$ is the diagonal mass matrix, $\mathbf{B} \in \mathbb{R}^{N \times 1}$ is the approximation of the control operator $B, \mathbf{B}_{d} \in \mathbb{R}^{N \times 1}$ is the approximation of the operator $B_{d}, \mathbf{v} \in \mathbb{R}^{N}$ is the vector of coordinates of $v_{h}$ in the finite element basis and $\mathbf{F}: \mathbb{R}^{N} \rightarrow \mathbb{R}^{N}$ stands for the nonlinear term in (4.1). Let us notice that using a finite difference method, we would have obtained a similar system. Substituting $v$ by $v_{h}$ in (4.2) and using the trapezoidal formula (4.7), we obtain the observation for the discrete system (4.8):

$$
\mathbf{y}_{o b s}=\mathbf{H v}+\eta \quad \text { with } \mathbf{H} \in \mathbb{R}^{3 \times N} \text { and } \eta=\left[\begin{array}{lll}
\eta_{1} & \eta_{2} & \eta_{3}
\end{array}\right]^{T} .
$$

For the numerical simulations, we shall simulate the measurement error $\eta(t)$ and the model error $\zeta(t)$ with the Matlab function 'randn' corresponding to uncorrelated zero-mean Gaussian random noises of covariance matrices $\mathbf{R}_{\eta}$ and $\mathbf{R}_{\zeta}$ respectively. We shall use these matrices to determine the filtering gain in the estimation equation.

\subsection{Feedback and filtering gains}

We find the feedback gain $K_{\omega}=-R^{-1} B^{*} P$ by solving the equation (2.8) in which we choose $Q=C^{*} C$ with $C=\left(-A_{0}\right)^{-1}$. We obtain $C v(t)=z(t)$ by solving the equation

$$
-\nu \Delta z(t)=v(t) \quad \text { in } \Omega, \quad \frac{\partial z(t)}{\partial n}=0 \quad \text { on } \Gamma_{n}, \quad z(t)=0 \quad \text { on } \Gamma_{d} .
$$

Here, we use the notation $K_{\omega}$ in place of $K$ as in Section 2 to emphasize that $K_{\omega}$ depends on the parameter $\omega \geq 0$ in equation (2.8). In the numerical tests, we shall compare the efficiency of different control laws for different values of this parameter $\omega$. Let us recall that the control space $U=\mathbb{R}$ is of dimension 1 . Thus $R>0$ is a real number. The operator $K$ satisfies the conclusions of Theorem 2.4. From the Riesz Theorem, we deduce that there exists a function $k_{\omega} \in L^{2}(\Omega)$, the kernel of the operator $K_{\omega}$, such that

$$
K_{\omega} v(t)=\int_{\Omega} k_{\omega}(x) v(x, t) \mathrm{d} x
$$

Next, the filtering operator $L_{\omega} \in \mathcal{L}\left(\mathbb{R}^{3}, L^{2}(\Omega)\right)$ is chosen of the form (2.11). The precise definition of $Q_{\mu}$ and $R_{\eta}$, or more precisely their discrete approximations used in the numerical tests, are given in Section 5.3 (see also Eq. (4.14) and the definition of the discrete approximation of $Q_{\mu}$ hereafter). If we denote by $\ell_{1, \omega}, \ell_{2, \omega}$ and $\ell_{3, \omega}$ the corresponding filtering gains, the compensator for system (4.1) can be written in the form

$$
\begin{aligned}
\frac{\partial v}{\partial t}= & \nu \Delta v-\left(\partial_{1} v+\partial_{2} v\right) w_{s}-\left(\partial_{1} w_{s}+\partial_{2} w_{s}\right) v-\left(\partial_{1} v+\partial_{2} v\right) v \quad \text { in } \Omega \times(0, \infty), \\
v(0)= & v_{0}+\mu_{0} \quad \text { in } \Omega, \\
\nu \frac{\partial v}{\partial n}= & 0 \quad \text { on } \Gamma_{n, 0} \times(0, \infty), \quad \nu \frac{\partial v}{\partial n}=h_{1}(x) \zeta(t) \quad \text { on } \Gamma_{n, \zeta} \times(0, \infty) \\
v(x, t)= & g_{1}(x) K_{\omega} v_{e}(t) \quad \text { on } \Gamma_{d} \times(0, \infty), \\
\frac{\partial v_{e}}{\partial t}= & \nu \Delta v_{e}-\left(\partial_{1} v_{e}+\partial_{2} v_{e}\right) w_{s}-\left(\partial_{1} w_{s}+\partial_{2} w_{s}\right) v_{e}, \\
& +\sum_{i=1}^{3} \ell_{i, \omega}\left(y_{e, i}-y_{o b s, i}\right) \quad \text { in } \Omega \times(0, \infty), \quad v_{e}(0)=v_{0} \quad \text { in } \Omega, \\
\nu \frac{\partial v_{e}}{\partial n}= & 0 \text { on } \Gamma_{n} \times(0, \infty), \quad v_{e}(x, t)=g_{1}(x) K_{\omega} v_{e}(t) \quad \text { on } \Gamma_{d} \times(0, \infty) .
\end{aligned}
$$

For the discrete system (4.8), the feedback gain $\mathbf{K}_{\omega} \in \mathbb{R}^{1 \times N}$ is defined by

$$
\mathbf{K}_{\omega}=-R^{-1} \mathbf{B}^{T} \mathbf{P}_{\omega} \mathbf{E}
$$


where $\mathbf{P}_{\omega}$ is the solution to the following Generalized Algebraic Riccati equation (GARE in brief)

$$
\begin{aligned}
& \mathbf{P}_{\omega} \in \mathbb{R}^{N \times N}, \mathbf{P}_{\omega}^{T}=\mathbf{P}_{\omega}, \mathbf{P}_{\omega} \geq 0, \\
& (\mathbf{A}+\omega \mathbf{E})^{T} \mathbf{P}_{\omega} \mathbf{E}+\mathbf{E P}_{\omega}(\mathbf{A}+\omega \mathbf{E})-\mathbf{E P}_{\omega} \mathbf{B} R^{-1} \mathbf{B}^{T} \mathbf{P}_{\omega} \mathbf{E}+\mathbf{C}^{T} \mathbf{E} \mathbf{C}=0,
\end{aligned}
$$

with $\mathbf{C}=-\mathbf{A}_{0}^{-1} \mathbf{E}$ and $\mathbf{A}_{0}$ is the stiffness matrix corresponding to equation (4.9). The operator $\mathbf{C}$ is the discrete approximation of the operator $C$. As the matrix $\mathbf{E}$ is diagonal and the matrix $-\mathbf{A}_{0}$ is a band matrix, their inversion are easily performed.

The filtering gain $\mathbf{L}_{\omega} \in \mathbb{R}^{N \times 3}$, the discrete approximation of $L_{\omega} \in \mathcal{L}\left(L^{2}(\Omega), \mathbb{R}^{3}\right)$, is defined by

$$
\mathbf{L}_{\omega}=-\mathbf{E P}_{e, \omega} \mathbf{H}^{T} \mathbf{R}_{\eta}^{-1},
$$

where $\mathbf{P}_{e, \omega}$ is the solution to the following Riccati equation

$$
\begin{aligned}
& \mathbf{P}_{e, \omega} \in \mathbb{R}^{N \times N}, \mathbf{P}_{e, \omega}^{T}=\mathbf{P}_{e, \omega}, \mathbf{P}_{e, \omega} \geq 0, \\
& (\mathbf{A}+\omega \mathbf{E}) \mathbf{P}_{e, \omega} \mathbf{E}+\mathbf{E} \mathbf{P}_{e, \omega}(\mathbf{A}+\omega \mathbf{E})^{T}-\mathbf{E} \mathbf{P}_{e, \omega} \mathbf{H}^{T} \mathbf{R}_{\eta}^{-1} \mathbf{H} \mathbf{P}_{e, \omega} \mathbf{E}+\mathbf{B}_{d} R_{\zeta} \mathbf{B}_{d}^{T}=0,
\end{aligned}
$$

the weight matrix $\mathbf{R}_{\eta}>0$ is the covariance of the noise $\eta$, and $R_{\zeta}>0$ is the variance of $\zeta$. $\left(\mathbf{R}_{\eta}>0\right.$ and $R_{\zeta}>0$ are assumed to be known.) Notice that, since the uncertainty $\zeta$ acts in a boundary condition, the matrix $\mathbf{B}_{d} R_{\zeta} \mathbf{B}_{d}^{T}$ plays the role of the discrete approximation of the operator $Q_{\mu}$ of equation (2.10).

The solution of the GAREs (4.12) and (4.14) are accomplished using a classical Schur decomposition of the corresponding Hamiltonian matrix [27]. The corresponding semi-discrete version of the compensator (4.10) is

$$
\begin{aligned}
\mathbf{E v}^{\prime} & =\mathbf{A} \mathbf{v}+\mathbf{B K}_{\omega} \mathbf{v}_{e}+\mathbf{F}(\mathbf{v})+\mathbf{B}_{d} \zeta, \quad \mathbf{v}(0)=\mathbf{v}_{0}+\mu_{0} \\
\mathbf{E v}_{e}^{\prime} & =\mathbf{A v}_{e}+\mathbf{L}_{\omega}\left(\mathbf{y}_{e}-\mathbf{y}_{o b s}\right)+\mathbf{B} \mathbf{K}_{\omega} \mathbf{v}_{e}, \quad \mathbf{v}_{e}(0)=\mathbf{v}_{0} \\
\mathbf{y}_{o b s} & =\mathbf{H v}+\eta, \quad \mathbf{y}_{e}=\mathbf{H v}_{e}
\end{aligned}
$$

where $\mathbf{v}_{0}$ is the coordinate vector of $v_{0 h}$ in the FEM basis.

\section{NumericAl EXPERIMENTS}

In this section, we present numerical results for the stabilization of system (4.15). To define the mesh size we set $n=60$, which corresponds to $N=3540$ degrees of freedom for the system (4.1). Throughout what follows, we choose

$$
\nu=\frac{1}{50} .
$$

\subsection{Determination of the unstable steady state}

Various unstable stationary solutions can be tested, see e.g. [8]. A very simple stationary solution is an affine function constant with respect to the $x_{1}$ variable, of the form $w_{s}\left(x_{1}, x_{2}\right)=a x_{2}+b$. The coefficients $a$ and $b$ are here determined from a series expansion of a tangent function. Our computation gives $a=-0.068$ and $b=0.0365$. This stationary solution is plotted in Figure 2 .

\subsection{Spectrum of the operator $(A, D(A))$}

To study the stability of linearized system associated to (4.1), we have to solve the discrete generalized eigenvalue problem corresponding to the system $A z=\lambda z,(\lambda, z) \in(\mathbb{C}, D(A) \backslash\{0\})$ :

$$
\begin{aligned}
& \nu \Delta z-\left(\partial_{1} z+\partial_{2} z\right) w_{s}-\left(\partial_{1} w_{s}+\partial_{2} w_{s}\right) z=\lambda z \quad \text { in } \Omega, \\
& \nu \frac{\partial z}{\partial n}=0 \quad \text { on } \Gamma_{n}, \quad z=0 \quad \text { on } \Gamma_{d} .
\end{aligned}
$$




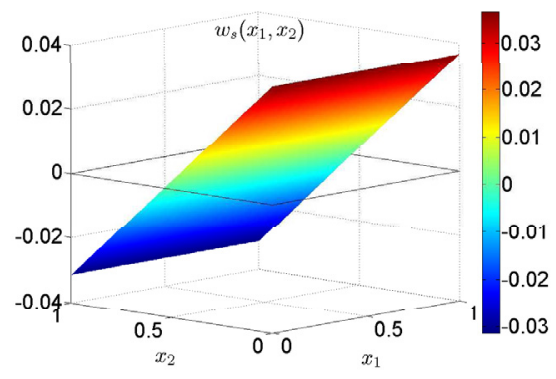

FiguRE 2. Unstable stationary solution.

TABle 1. Partial spectrum of (E, A).

\begin{tabular}{cccccc}
\hline$i$ & 1 & 2 & 3 & 4 & 5 \\
\hline$\lambda_{i}$ & 0.017 & -0.184 & -0.383 & -0.588 & -0.771 \\
\hline
\end{tabular}
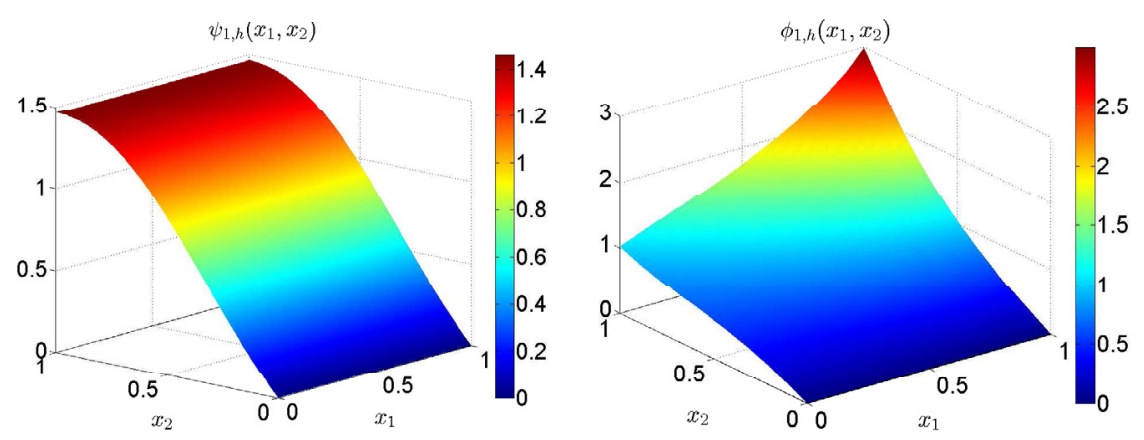

FigURE 3. Graph of a normalized right unstable eigenvector $\psi_{1, h}$ and left unstable eigenvector $\phi_{1, h}$ of $(\mathbf{E}, \mathbf{A})$.

From a numerical view point, we look for $(\mathbf{z}, \lambda) \in(\mathbb{C} \backslash\{0\})^{n} \times \mathbb{C}$ solution of

$$
\mathbf{A z}=\lambda \mathbf{E z} .
$$

A part of the spectrum of $(\mathbf{E}, \mathbf{A})$ is computed numerically with the procedure eigs in Matlab. The five eigenvalues close to the imaginary axis are given in Table 1 . We notice that only one eigenvalue of the pair $(\mathbf{E}, \mathbf{A})$ is with non negative real part. The eigenfunction $\psi_{1}$ associated with $\lambda_{1}$ only depends on $x_{2}$. The approximate eigenfunctions $\psi_{1, h}$ and $\psi_{2, h}$ corresponding to respectively to $\lambda_{1}$ and $\lambda_{2}$ are represented in Figures 3 and 4 .

\subsection{Computation of functional gains}

We have to verify that $\left(H_{4}\right)$ and $\left(H_{5}\right)$ are satisfied for some $\omega \geq 0$. If we choose $\omega=0.078$, the only unstable eigenvalues of $A+\omega I$ are $\left(\lambda_{i}+\omega\right)_{1 \leq i \leq 5}$ where $\left(\lambda_{i}\right)_{1 \leq i \leq 5}$ are the eigenvalues in Table 1 . These eigenvalues are simple. Let us denote by $\left(\psi_{i}\right)_{1 \leq i \leq 5}$ the corresponding eigenfunctions of $A$ and by $\left(\phi_{i}\right)_{1 \leq i \leq 5}$ the corresponding eigenfunctions of $A^{*}$ chosen in such a way that the two families are bi-orthogonal.

To verify assumption $\left(H_{4}\right)$, we have to compute

$$
\mathcal{B}_{i}:=B^{*} \phi_{i}=-\nu \int_{\Gamma_{c}} g_{1} \frac{\partial \phi_{i}}{\partial n}=\nu \int_{0}^{1} \sin \left(\pi x_{1}\right) \frac{\partial \phi_{i}}{\partial x_{2}}\left(x_{1}, 0\right) \mathrm{d} x_{1},
$$

for $1 \leq i \leq 5$. 

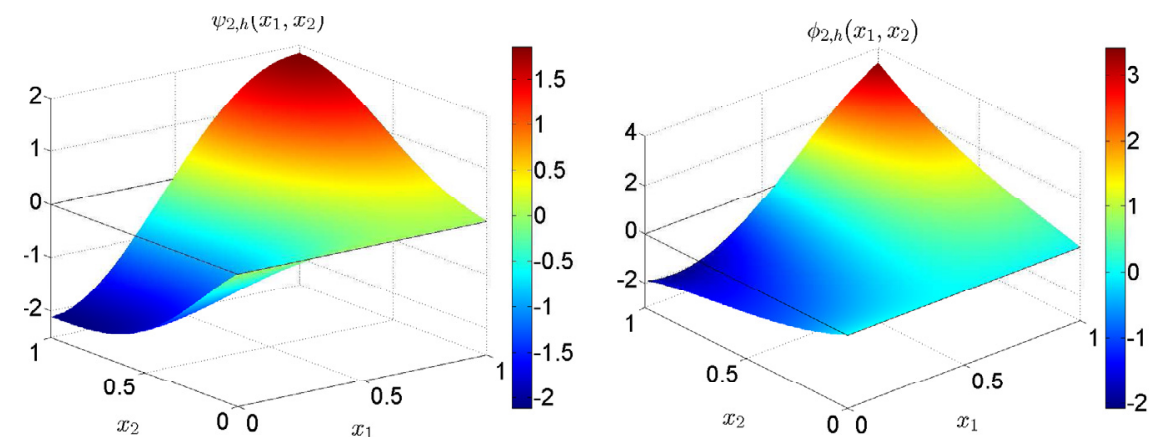

FiguRE 4. Graph of a normalized right stable eigenvector $\psi_{2, h}$ and left stable eigenvector $\phi_{2, h}$ of $(\mathbf{E}, \mathbf{A})$.

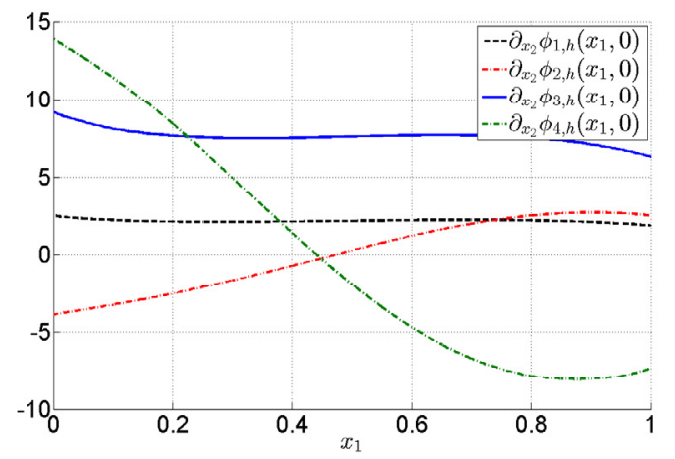

FiguRE 5. Graph of a normalized normal derivative of four left eigenvectors $\phi_{i, h}$ at the boundary $\Gamma_{d}$.

To verify assumption $\left(H_{5}\right)$, we have to compute

$$
H \psi_{i}=\left(\frac{1}{0.05} \int_{0.2}^{0.25} \psi_{i}\left(x_{1}, 1\right) \mathrm{d} x_{1}, \frac{1}{0.05} \int_{0.5}^{0.55} \psi_{i}\left(x_{1}, 1\right) \mathrm{d} x_{1}, \frac{1}{0.05} \int_{0.8}^{0.85} \psi_{i}\left(x_{1}, 1\right) \mathrm{d} x_{1}\right),
$$

for $1 \leq i \leq 5$. We set $\mathcal{H}_{i}:=\left|H \psi_{i}\right|$, where $\left|H \psi_{i}\right|$ stands for the Euclidian norm of the vector $H \psi_{i} \in \mathbb{R}^{3}$. The values of $\left|\mathcal{B}_{i}\right|$ and $\mathcal{H}_{i}$ are reported below

\begin{tabular}{|c|c|c|c|c|}
\hline & 0.02760 .0016 & 0.096 & 0.00 & 0138 \\
\hline $\mathcal{H}_{i}$ & $\begin{array}{ll}2.60 & 2.38 \\
\end{array}$ & 2.25 & 2.3 & 2.40 \\
\hline
\end{tabular}

As all coefficients are different from 0 , we conclude that, for all $0<\omega \leq 0.078$, the pair $(A+\omega I, B)$ is stabilizable and the pair $(A+\omega I, H)$ is detectable. We notice that the second and the fourth mode are less stabilizable than the other ones, because $\left|\mathcal{B}_{2}\right|$ and $\left|\mathcal{B}_{4}\right|$ are much smaller than $\left|\mathcal{B}_{1}\right|$ and $\left|\mathcal{B}_{3}\right|$. To keep a control force in acceptable values, we will choose two different values of $\omega$, namely $\omega_{0}=0$ and $\omega_{1}=0.21$, which corresponds to a value satisfying $\lambda_{3}<-\omega_{1}<\lambda_{2}$. The choice of having only one actuator with the function $g_{1}(x)=\sin \left(\pi x_{1}\right)$ is not well adapted to stabilize the modes number 2 and 4 (see Fig. 5). If we would like to improve the efficiency of the control law, we have to increase the dimension of the control space and to choose other actuators $\left(g_{i}\right)_{2 \leq i \leq N_{c}}$ appropriately.

In the numerical simulations, we shall test two values for $\omega$, namely

$$
\omega_{0}=0 \quad \text { and } \quad \omega_{1}=0.21 \text {. }
$$



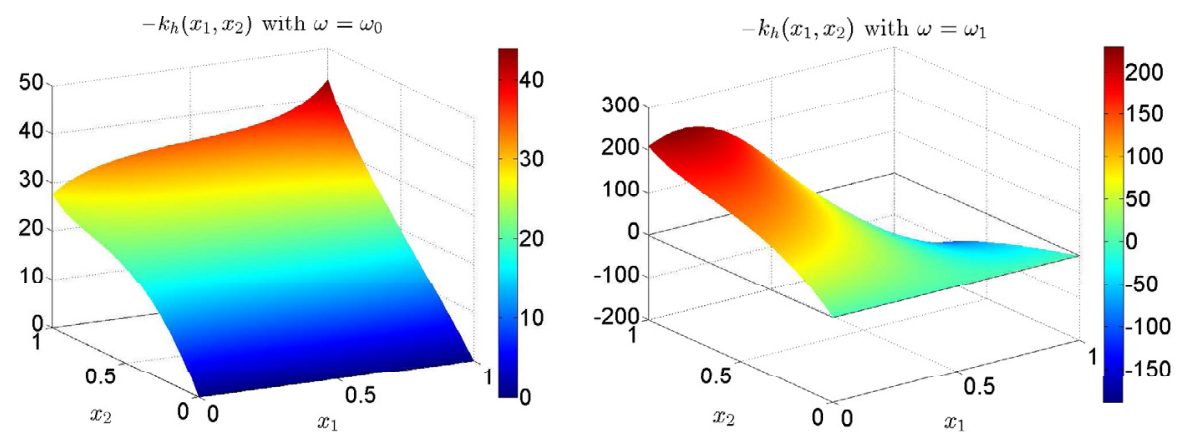

Figure 6. Finite element approximation of functional gain $-k$ for $R=1$ and $\omega=\omega_{0}$ (left), $\omega=\omega_{1}$ (right).
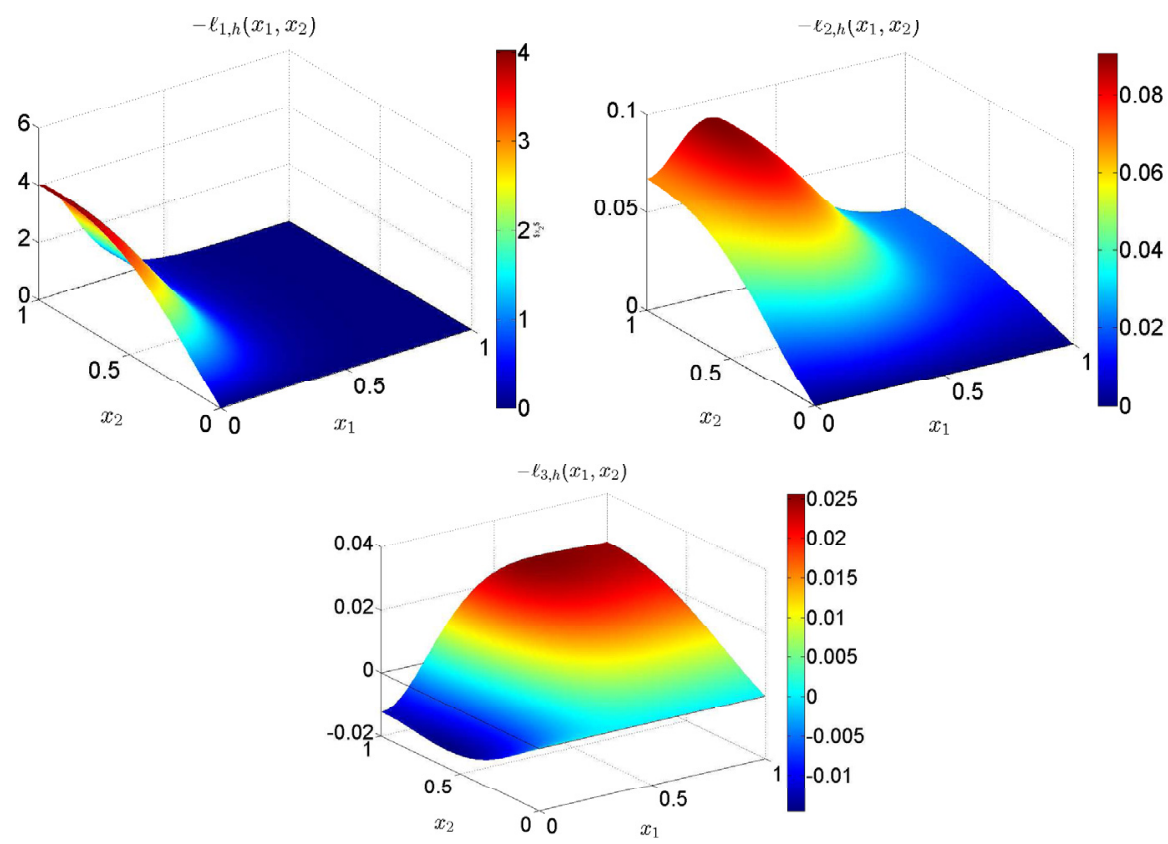

FIGURE 7. Finite element approximations of functional filter gains for $\omega=0, R_{\eta}=4 \times 10^{-6} I_{3}$ and $R_{\zeta}=4 \times 10^{-6}$.

To obtain a fast stabilization of the closed-loop system, we set $R=1$ in (4.12). This parameter is sufficiently small to allow large control amplitudes. From the expression of the feedback gain $\mathbf{K}_{\omega}$ in (4.11), we deduce the coordinates of the finite element approximation $k_{\omega, h}$ of the functional gain $k_{\omega}$. In Figure 6 , we have plotted the graph of $k_{\omega, h}$ with $\omega=\omega_{0}$ and $\omega=\omega_{1}$. For $\omega_{0}$, we notice that $k_{\omega, h}$ has the same behaviour as the adjoint unstable eigenvector $\psi_{1}$ of $A$. By increasing the dimension of the unstable subspace with a shift $\omega$ equal to $\omega_{1}$, we increase dramatically the control force. In Figure 6 (right), we have plotted the feedback gain $k_{\omega, h}$ computed with $A+w_{1} I$ in (4.12). Note that the graph of the feedback gain is now very similar to the graph of $\psi_{2}$. It is the second mode that contributes the most significantly to the feedback stabilization.

The covariance matrix of measurements is $\mathbf{R}_{\eta}=4 \times 10^{-6} \mathbf{I}_{3}$ and $R_{\zeta}=4 \times 10^{-6}$. In Figure 7 , the three kernels of $L_{\omega}$ are plotted with $\omega=\omega_{0}$. We notice that the values of $\ell_{1, \omega, h}$ are globally greater than those of $\ell_{2, \omega, h}$ and $\ell_{3, \omega, h}$ and more particularly close to $\{0\} \times(0,1)$. Recall that these functions are involved in the 

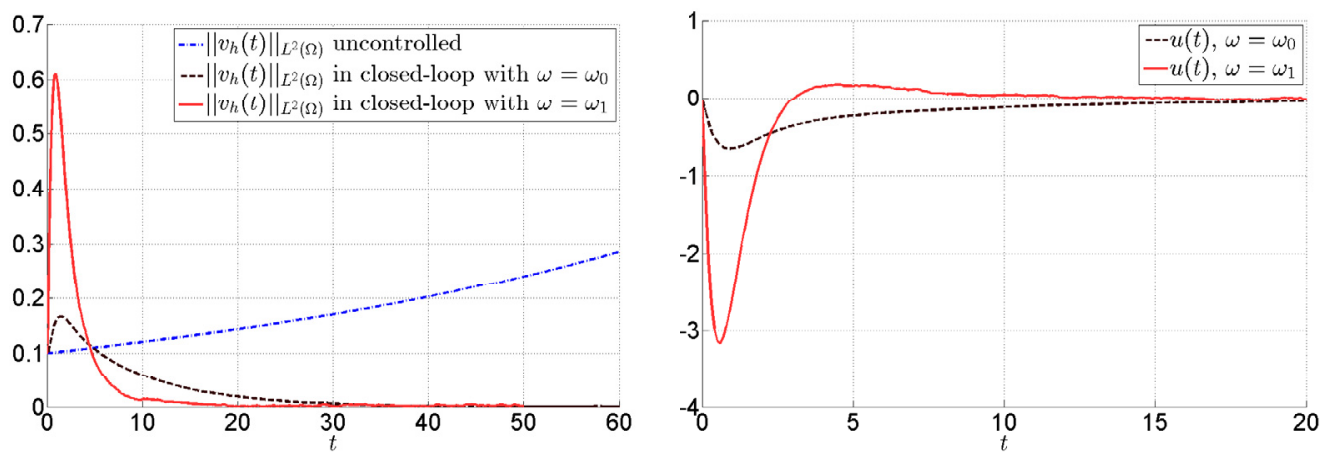

Figure 8. (Left) Evolution of $\left\|v_{h}(t)\right\|_{2}$ with respect to $t$ for the linear uncontrolled and closedloop systems with $\omega=\omega_{0}$ and $\omega=\omega_{1}$. (Right) Evolution of corresponding control laws for the linear closed-loop system.

differential equation (4.10) for the estimation $w_{e}$ of $w$, in the expression $\sum_{i=1}^{3} \ell_{i, \omega}\left(y_{e, i}-y_{o b s, i}\right)$. Consequently, a large difference between $y_{o b s, 1}(t)$ and $y_{e, 1}(t)$ will have an impact on the solution $w_{e}$ larger than that of $\left(y_{e, 2}(t)-y_{o b s, 2}(t)\right)$ or $\left(y_{e, 3}(t)-y_{o b s, 3}(t)\right)$.

\subsection{Numerical tests for the linearized system}

First, we present the results for the integration of the linear closed-loop system associated to problem (4.15) in which we set $\mathbf{F}=0$. We choose the initial condition $v_{0}=0$ and $\mu_{0}$ as an eigenfunction corresponding to the unstable eigenvalue of $A$

$$
\mu_{0}=\delta \psi_{1}
$$

where $\delta>0$ is used as a parameter to vary the magnitude of $\mu_{0}$. In practice, we take $\delta=0.1$. The time integration is performed with the BDF scheme of order 2 [35]. The system (4.15) is solved up to $T=100$, a choice large enough to capture the effect of both feedback and filtering operators in the nonlinear coupled system. On the left hand side in Figure 8, we have represented the $L^{2}$ norm of the solutions $v_{h}(t)$ for the linear uncontrolled and controlled systems. We see that, after an initial increase of the norm, the norm of the solution to closed-loop system decreases quickly to 0 . As expected, the decay rate is higher with $\omega_{1}$ than with $\omega_{0}$. The uncontrolled solution explodes exponentially. On the right hand side in Figure 8, we have plotted the control $u$. It shows a control acting strongly close to $t=0$ and then oscillating around zero after $t=20$.

In order to better understand the role of the filtering operator in the estimation process, we have represented in Figure 9 the evolution of the three measurements with respect to time. We recall that $y_{o b s, 1}, y_{o b s, 2}$ and $y_{o b s, 3}$ respectively correspond to the noisy measurements of $v$ in $(0.2,0.25) \times\{1\},(0.5,0.55) \times\{1\}$ and $(0.8,0.85) \times\{1\}$. As expected, we can see that the estimated measurements are much smoother than the noisy measurements. Due to the large gain of $\ell_{1, \omega}$, we notice that the estimated measurement $y_{e, 1}$ converges rapidly to the noisy measurement $y_{o b s, 1}$. Notice that the convergence is faster when $\omega=\omega_{1}$.

\subsection{Numerical tests for the nonlinear system}

Now we deal with the fully nonlinear system (4.15). Such a system evolves differently from the linear one. As in the linear case, the numerical stabilization tests are done with $\delta=0.1$. In this case, the linearized system is a good approximation of the nonlinear one.

In Figure 10, on the left, we have represented the $L^{2}$ norm of the solution $v_{h}(t)$ for the nonlinear uncontrolled and controlled systems (4.10). For $\omega=\omega_{0}$, the solution behaves in the same way as in the linear case. In the case where $\omega=\omega_{1}$, the nonlinear terms become more significant and the approximation of the nonlinear system by the linear one is less justified. The stabilization results show that the nonlinear controlled system with $\omega=\omega_{1}$ 

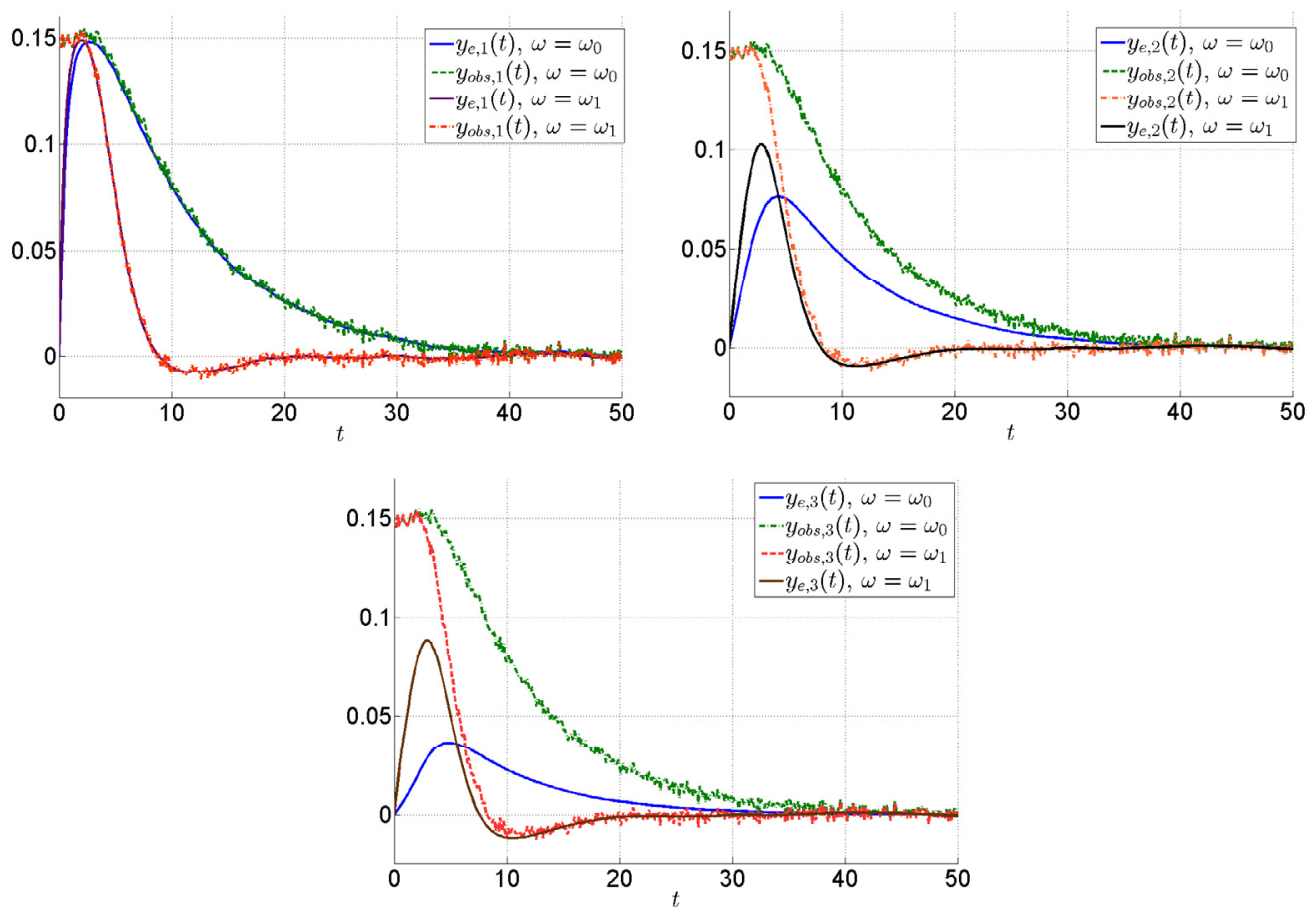

Figure 9. Evolution of the controlled and estimated measurements for the linear closed-loop system satisfied by $w_{h}$ when $\omega=\omega_{0}$ and $\omega=\omega_{1}$.
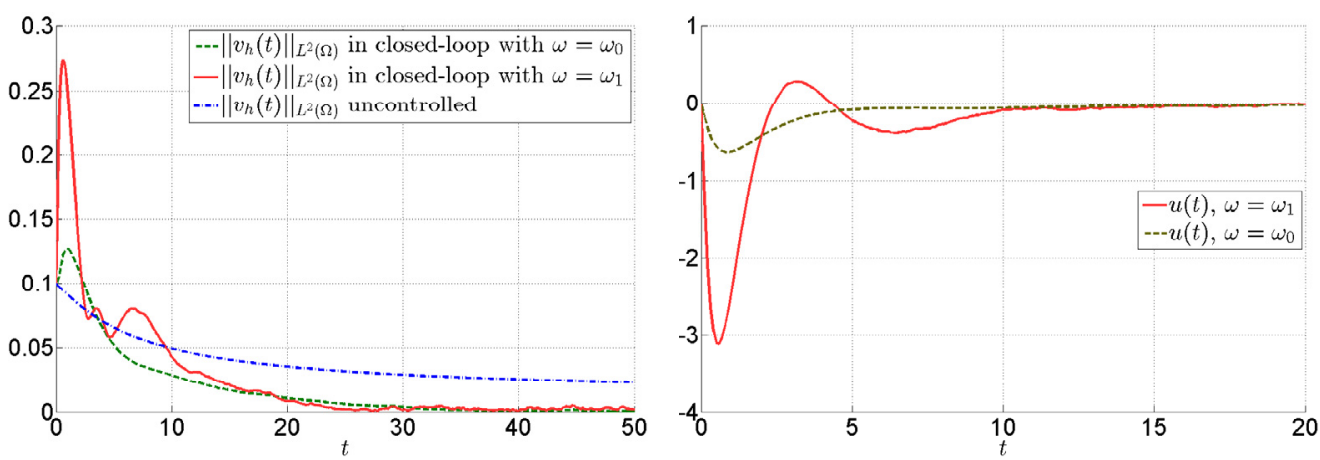

Figure 10. (Left) Evolution of the $L^{2}$ norm of the solution $w_{h}(t)$ for the nonlinear closedloop system and uncontrolled system. (Right) Evolution of the control laws for the nonlinear closed-loop system.

is not better than the one controlled with $\omega=\omega_{0}$. These results illustrate how the linear controller can locally stabilize the nonlinear system when only partial observations are available. However, the choice of actuators and sensors become fundamental to correctly stabilizes the nonlinear system.

To compare the quality of the estimation when we replace the linear system by the nonlinear one, we have plotted in Figure 11 the time evolution of the noisy and estimated observations. We can verify that, as in the linear case, the effect of the noise is highly reduced by the estimation process but the convergence of the estimated observation towards the noisy observation is slower than in the linear case. In Figure 12, the nonlinear 

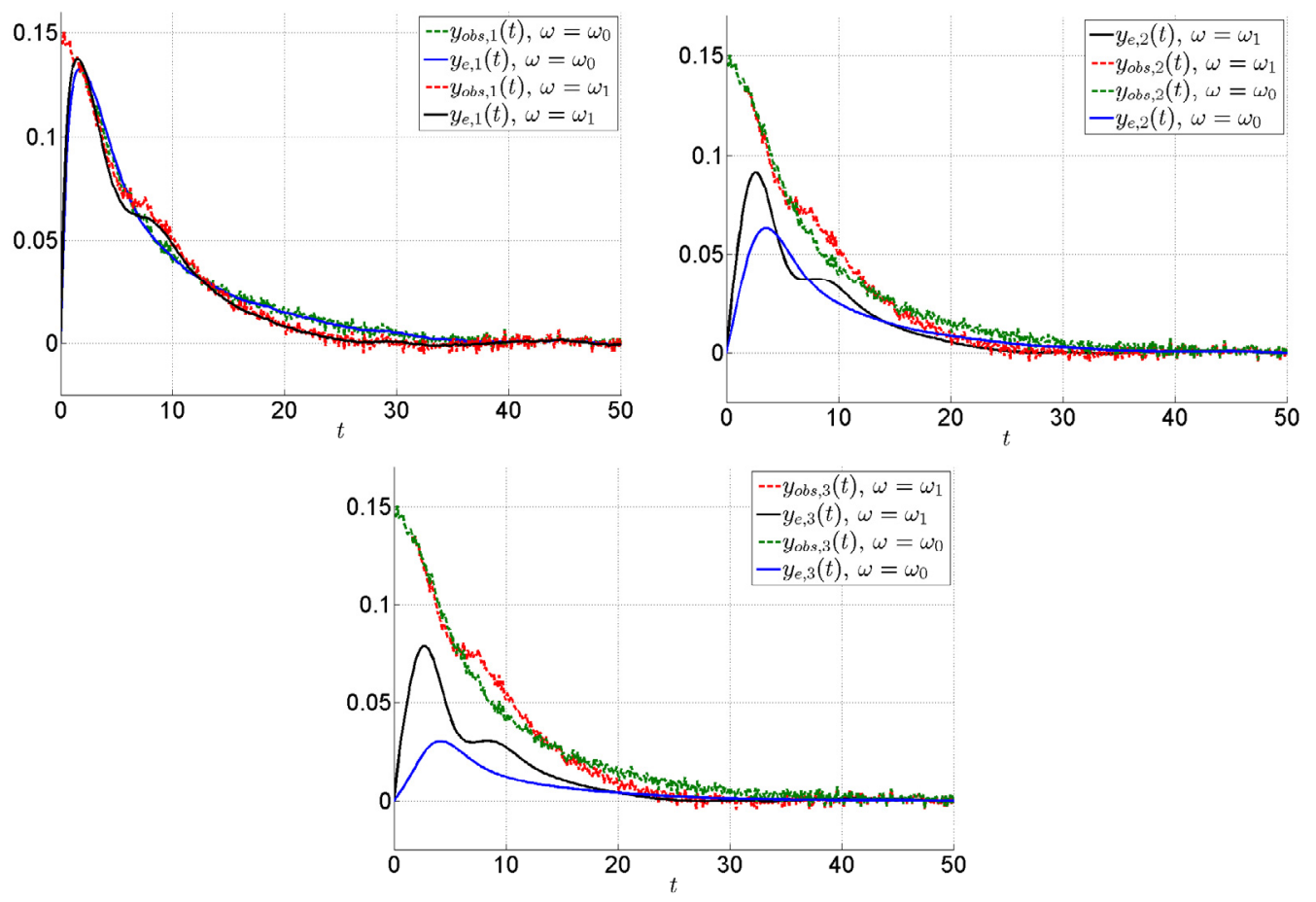

FIGURE 11. Evolution of the controlled and estimated measurements for the nonlinear closedloop system satisfied by $w_{h}$.
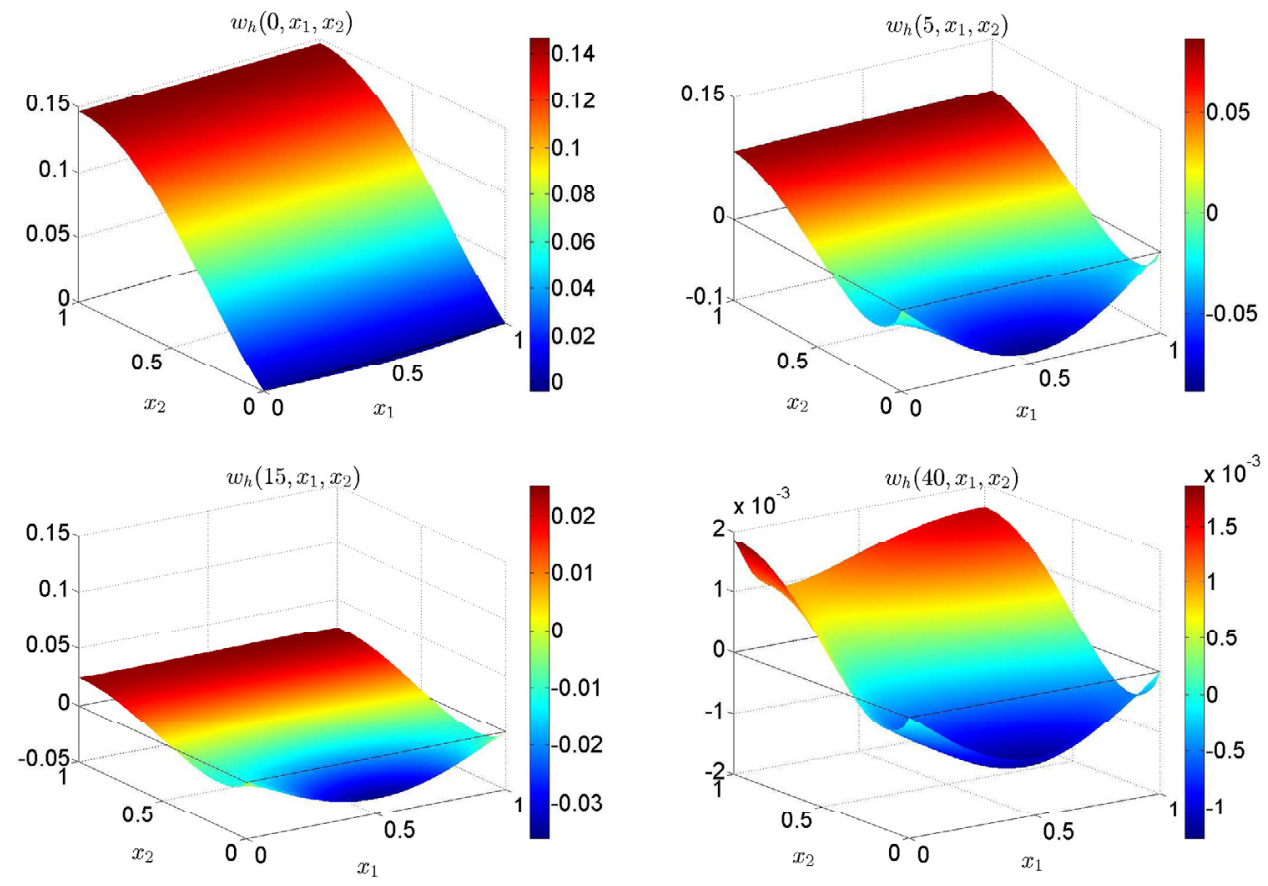

FigURE 12. Evolution of the solution $v_{h}$ for the nonlinear system in closed-loop (4.15) at times $t=0, t=5, t=15$ and $t=40$. 
closed-loop solution $v_{h}$ is represented for four different times. We can check that the stabilization is achieved up to small oscillations on the boundaries where the control and the model noise are acting.

\section{Conclusion}

In this paper, we studied theoretically and numerically the boundary stabilization of a Burger's equation, locally around an unstable stationary solution, in presence of measurement and model errors. A Luenberger compensator is built from the linearized Burgers' equation. The feedback gains are obtained by solving an algebraic Riccati equation. In Sections 2 and 3, we give some assumptions on the control and observation operators that allows us to study the well posedness of the infinite dimensional compensator. At the end of Section 3, we verify on some particular examples whether these assumptions are satisfied or not. In Section 4, we prove that the controlled nonlinear Burgers equation coupled with the linear estimator can be locally stabilized. In Section 5 , the numerical results illustrate these theoretical results: The solution estimated from noisy measurements may be used into a feedback law to stabilize efficiently the nonlinear unstable system, when the initial data and the boundary perturbation are not too large.

\section{REFERENCES}

[1] M. Badra, Lyapunov function and local feedback boundary stabilization of the Navier-Stokes equations. SIAM J. Control Optim. 48 (2009) 1797-1830.

[2] M. Badra, Abstract settings for stabilization of nonlinear parabolic system with a Riccati-based strategy. Application to NavierStokes and Boussinesq equations with Neumann or Dirichlet control. Discrete Contin. Dyn. Syst. 32 (2012) 1169-1208.

[3] M. Badra and T. Takahashi, Stabilization of parabolic nonlinear systems with finite dimensional feedback or dynamical controllers: application to the Navier-Stokes system. SIAM J. Control Optim. 49 (2011) 420-463.

[4] H.T. Banks, R.C. Smith and Y. Wang, Smart Material Structures, modeling, estimation and control. Masson/John Wiley, Paris/Chichester (1996).

[5] A. Bensoussan, G. Da Prato, M.C. Delfour and S.K. Mitter, Representation and Control of Infinite Dimensional Systems. Vol. 1. Birkhäuser, Boston (1992).

[6] A. Bensoussan, G. Da Prato, M.C. Delfour and S.K. Mitter, Representation and Control of Infinite Dimensional Systems. Vol. 2. Birkhäuser, Boston (1992).

[7] J. Burns and H. Marrekchi, Optimal fixed-finite-dimensional compensator for Burgers' equation with unbounded input/output operators, Computation and Control III. Progress System Control Theory. Vol. 15. Birkhauser, Boston, MA (1993) 83-104.

[8] J. Burns, A. Balogh, D.S. Gilliam and V.I. Shubov, Numerical Stationary Solutions for a Viscous Burgers' Equation. J. Math. Syst. Estim. Control 8 (1998) 1-16.

[9] C.M. Chen and V. Thomée, The lumped mass finite element method for a parabolic problem. J. Austral. Math. Soc. Ser. B 26 (1985) 329-354.

[10] J.-M. Coron, Control and nonlinearity. American Mathematical Society. Providence, RI (2007).

[11] R. Curtain, Finite dimensional compensators for parabolic distributed systems with unbounded control and observation. SIAM J. Control Optim. 22 (1984) 255-276.

[12] R. Curtain, A comparison of finite-dimensional controller designs for distributed parameter systems, Control Theory Adv. Technol. 9 (1993) 609-628.

[13] R. Curtain and D. Salamon, Finite dimensional compensators for infinite dimensional systems with unbounded input operators. SIAM J. Control Optim. 24 (1986) 797-816.

[14] R. Curtain and H.J. Zwart, An introduction to infinite dimensional linear systems theory. Springer-Verlag, New York (1995).

[15] H.C. Elman, D.J. Silvester and A.J. Wathen, Finite Elements and Fast Iterative Solvers: with Applications in Incompressible Fluid Dynamics. Oxford University Press, New York (2005).

[16] A.V. Fursikov, Optimal control of distributed systems. Theory and Applications. AMS Providence, Rhode Island (2000).

[17] P. Grisvard, Caractérisation de quekques espaces d'interpolation. Arch. Rat. Mech. An. 25 (1967) $40-63$.

[18] P. Grisvard, Elliptic problems in nonsmooth domains. Pitman Monogr. Studies in Mathematics. Vol. 24. Advanced Publishing Program, Boston, MA (1985).

[19] P. Grisvard, Singularities in boundary value problems. Recherches en Mathématiques Appliquées [Research in Applied Mathematics]. Vol. 22. Masson, Paris; Springer-Verlag, Berlin (1992).

[20] G. Ji and I. Lasiecka, Partially observed analytic systems with fully unbounded actuators and sensors-FEM algorithms. Comput. Optim. Appl. 11 (1998) 111-136.

[21] S. Jund and S. Salmon, Arbitrary high-order finite element schemes and high-order mass lumping. Int. J. Appl. Math. Comput. Sci. 17 (2007) 375-393.

[22] T. Kato, Perturbation theory for linear operators, Reprint of the 1980 Edition. Springer-Verlag (1995).

[23] S. Kesavan and J.P. Raymond, On a degenerate Riccati equation. Control Cybern. 38 (2009) 1393-1410. 
[24] I. Lasiecka, Galerkin approximations of infinite dimensional compensators for flexible structures with unbounded control action. Acta Appl. Math. 28 (1992) 101-133.

[25] I. Lasiecka, Finite element approximations of compensator design for analytic generators with fully unbound controls/observations. SIAM J. Control Optim. 33 (1995) 67-88.

[26] I. Lasiecka and R. Triggiani, Control Theory for Partial Differential Equations: Continuous and Approximations Theories. Vol. I. Cambridge University Press, Cambridge (2000).

[27] A.J. Laub, A Schur method for solving algebraic Riccati equations. IEEE Trans. Automat. Control 24 (1979) $913-925$.

[28] J.-L. Lions, Contrôle optimal des équations aux dérivées partielles. Dunod, Paris (1968).

[29] J.-L. Lions and E. Magenes, Non-homogeneous boundary value problems and applications. Vol. 2. Springer, Berlin-HeidelbergNew York (1972).

[30] A. Pazy, Semigroups of linear operators and applications to partial differential equations. Springer-Verlag, New-York (1983).

[31] J.P. Raymond, Boundary feedback stabilization of the two dimensional Navier-Stokes equations. SIAM J. Control Optim. 45 (2006) 790-828.

[32] J.P. Raymond, Feedback boundary stabilization of the three dimensional incompressible Navier-Stokes equations. J. Math. Pures Appl. 87 (2007) 627-669.

[33] J.P. Raymond, Stabilizability of infinite dimensional systems by finite dimensional controls, submitted.

[34] J.-P. Raymond and L. Thevenet, Boundary feedback stabilization of the two dimensional Navier-Stokes equations with finite dimensional controllers. Discrete Contin. Dyn. Syst. 27 (2010) 1159-1187.

[35] L.F. Shampine, I. Gladwell and S. Thompson, Solving ODEs with MATLAB. Cambridge University Press, Cambridge (2003).

[36] J.M. Schumacher, A direct approach to compensator design for distributed parameter systems. SIAM J. Control and Optim. 21 (1983) 823-836.

[37] L. Thevenet, Lois de feedback pour le contrôle d'écoulements. Ph.D. Thesis, Université de Toulouse (2009).

[38] L. Thevenet, J.-M. Buchot and J.-P. Raymond, Nonlinear feedback stabilization of a two-dimensional Burgers equation. ESAIM: COCV 16 (2010) 929-955.

[39] M. Tucsnak and G. Weiss, Observation and control for operator semigroups. Birkhuser Verlag, Basel (2009). 